\title{
Towards measuring growth rates of pathogens during infections by $\mathrm{D}_{2} \mathrm{O}$-labeling lipidomics
}

Cajetan Neubauer ${ }^{1,2, *}$, Alex L. Sessions ${ }^{2}$, Ian R. Booth ${ }^{4}$, Benjamin P. Bowen ${ }^{5}$, Sebastian H. Kopf $^{6}$, Dianne K. Newman ${ }^{1,2}$, Nathan F. Dalleska ${ }^{3}$

1. Division of Biology and Biological Engineering, California Institute of Technology, Pasadena,

CA 91125, USA

2. Division of Geological and Planetary Sciences, California Institute of Technology

3. Environmental Analysis Center, California Institute of Technology

4. Institute of Medical Sciences, University of Aberdeen, Aberdeen AB25 2ZD, UK

5. DOE Joint Genome Institute, Walnut Creek, CA 94598, USA

6. Department of Geological Sciences, University of Colorado, Boulder, CO 80309, USA

$\S$ IRB was a CEMI-funded visiting research fellow at Caltech and Leverhulme Emeritus Research Fellow during this study.

*To whom correspondence should be addressed:

E-mail: caj@caltech.edu

15 Keywords: Lipidomics, Isotope-ratio mass spectrometry, Microbial growth rate, Stable-isotope probing, Heavy water 
ABSTRACT

RATIONALE: Microbial growth rate is an important physiological parameter that is challenging to measure in situ, partly because microbes grow slowly in many environments. Recently, it has been demonstrated that generation times of S. aureus in cystic fibrosis (CF) infections can be determined by $\mathrm{D}_{2} \mathrm{O}$-labeling of actively synthesized fatty acids. To improve species specificity and allow growth rate monitoring for a greater range of pathogens during the treatment of infections, it is desirable to accurately quantify trace incorporation of deuterium into phospholipids.

METHODS: Lipid extracts of $\mathrm{D}_{2} \mathrm{O}$-treated E. coli cultures were measured on LC-ESI-MS instruments equipped with TOF and Orbitrap mass analyzers, and used for comparison with the analysis of fatty acids by isotope-ratio GC-MS. We then develop an approach to enable tracking of lipid labeling, by following the transition from stationary into exponential growth in pure cultures. Lastly, we apply $\mathrm{D}_{2} \mathrm{O}$-labeling lipidomics to clinical samples from $\mathrm{CF}$ patients with chronic lung infections.

RESULTS: Lipidomics facilitates deuterium quantification in lipids at levels that are useful for many labeling applications $(>0.03$ at $\%$ D). In the E. coli cultures, labeling dynamics of phospholipids depend largely on their acyl chains and between phospholipids we notice differences that are not obvious from absolute concentrations alone. For example, cyclopropyl-containing lipids reflect the regulation of cyclopropane fatty acid synthase, which is predominantly expressed at the beginning of stationary phase. The deuterium incorporation into a lipid that is specific for $S$. aureus in CF sputum, indicates an average generation time of the pathogen on the order of one cell doubling per day.

CONCLUSIONS: This study demonstrates how trace level measurement of stable isotopes in intact lipids can be used to quantify lipid metabolism in pure cultures and provides guidelines that enable growth rate measurements in microbiome samples after incubation with a low percentage of $\mathrm{D}_{2} \mathrm{O}$. 


\section{INTRODUCTION}

Bacteria continually react to diverse stimuli, such as the availability of nutrients and electron acceptors, exposure to antimicrobial drugs or attack by the immune system. However, measuring microbial metabolites and growth rates within a complex environment still poses many technical challenges. Two recent advances in microbial ecology are beginning to make measuring average growth rates in environmental samples possible. The first advance is based on metagenomic DNA sequencing and takes advantage of the observation that growing cells yield more sequencing reads at genomic regions near the origin of replication ${ }^{[1,2]}$. This method is applicable to any microbial species in a microbiome as long as its assembled genome has a high sequence coverage. The second advance uses isotopic labeling to determine the biosynthesis rates of microbial lipid metabolites by mass spectrometry ${ }^{[3,4]}$. Stable-isotope probing has a larger dynamic range than sequencing and can be used to quantify the slow growth rates that microbes have under environmental conditions. A limitation of stable-isotope probing, however, is the identification of metabolites that are diagnostic for a specific microorganism. It is therefore desirable to combine isotopic labeling with a method such as lipidomics, which can detect a large number of microbial metabolites.

Lipids have been used for decades in ecology as markers of microbial metabolism, where they reveal information about viable biomass, nutritional status or changes of the microbial community structure ${ }^{[5,6]}$. Also, lipids can still be analyzed long after nucleic acids and peptides are degraded ${ }^{[7]}$. In order to estimate the growth rates of microbes, the active production of strain- and genus-specific lipid metabolites can be measured with stable isotope labeling ${ }^{[3]}$. With advances in soft-ionization mass spectrometry we can now attempt to combine isotope quantification and lipidomics for the study of microbes in situ.

Soft-ionization mass spectrometry detects thousands of lipids in environmental extracts and would in principle be well-suited to quantify the biosynthesis of lipid biomarkers by itself ${ }^{[8-11]}$. However, extraction yields and ionization efficiency vary widely between samples ${ }^{[12-16]}$. This 
currently poses severe constraints on measuring lipid production rates and effectively limits estimating bacterial growth rates in natural environments.

Ratios of isotopes can be measured with high accuracy by mass spectrometry, partly because ratiometric readouts vary less than absolute ion intensities ${ }^{[17]}$. For lipid biosynthesis, the incorporation of an isotope tracer such as ${ }^{13} \mathrm{C}$-labeled substrates hence can provide a robust way to quantify anabolic activity and lipid turnover ${ }^{[18,19]}$. In microbiome samples bacteria differ widely in their ability to take up carbon sources and gases such as $\mathrm{CO}_{2}$, depending on their genetic capabilities and metabolic states. $\mathrm{D}_{2} \mathrm{O}$ is a non-discriminating tracer of de novo lipid biosynthesis and thus often better suited for microbiome studies. $\mathrm{D}_{2} \mathrm{O}$-labeling has recently been used to estimate in situ growth rates of Staphylococcus aureus in chronically infected lungs. After labeling of expectorated sputum with $\mathrm{D}_{2} \mathrm{O}$, the deuterium enrichment of anteiso fatty acids was quantified using GC pyrolysis isotope-ratio $\mathrm{MS}(\mathrm{GC} / \mathrm{P} / \mathrm{IRMS})$ in order to estimate the growth rate of the pathogen ${ }^{[3]}$. Previous studies have been used to study lipid biosynthesis with $\mathrm{D}_{2} \mathrm{O}$ in vivo ${ }^{[20-25]}$. Environmental samples, however, provide particular challenges. For example, microbes often grow slowly in situ and one can expect low rates of deuterium incorporation into lipids ${ }^{[3]}$. Whether trace levels of incorporation can reliably be detected remains to be studied before deuterium incorporation can be used to determine lipid biosynthesis rates in situ by lipidomics.

In this study we apply stable isotope probing with $\mathrm{D}_{2} \mathrm{O}$ and measure deuterium incorporation by MS-based lipidomics. This approach can be used to obtain labeling rates for individual intact lipids in environmental samples, where microorganisms typically grow slowly. We begin by characterising several technical aspects that have to do with quantifying low levels of deuterium labeling. We then refine our application of $\mathrm{D}_{2} \mathrm{O}$-labeling lipidomics by tracking of lipid labeling in E. coli during the stationary-to-log phase transition. This reveals lipids that have distinct labeling dynamics that are not obvious from measuring absolute analyte concentrations alone. Lastly, we test $\mathrm{D}_{2} \mathrm{O}$-labeling lipidomics in a clinical context with the aim to measure the growth of 
S. aureus in cystic fibrosis lung infections. In sum this study establishes principles for how growth rates of microbes in situ can be estimated by stable isotope probing lipidomics.

\section{RESULTS}

When cells grow after addition of heavy water, newly synthesized biomass will contain more D.

This also means that each lipid pool will be a mixture of molecules that vary more in their D abundance. The introduced heterogeneity causes broadening of chromatographic peaks, which could skew the isotope ratio observed by LC-MS as ionization efficiency varies over time ${ }^{[26,27]}$. In order to evaluate how this affects isotope quantification by lipidomics, we grew an E. coli culture in $4 \% \mathrm{D}_{2} \mathrm{O}$ (fractional D-abundance, ${ }^{2} F_{\text {WATER }}$ ) and measured lipids after chromatographic separation using an ESI-TOF mass spectrometer ${ }^{[28]}$.

E. coli has a comparatively simple lipid composition and its lipid metabolism has been studied for decades ${ }^{[29]}$. The bacterium therefore provides a solid model system to develop and test methods for stable isotope labeling lipidomics. E. coli lipid extracts contains mainly phosphatidylethanolamines (PE), phosphatidylglycerol (PG) and cardiolipins (CL) ${ }^{[30]}$. When fully labeled in $4 \% \mathrm{D}_{2} \mathrm{O}$, the molecular ions from PE and PG lipids extend over a range of $8 \mathrm{~m} / z$. As expected, labeling causes a shift of retention time (Figure 1A). Strongly deuterated molecules elute earlier than lighter ones (Figure 1B). The maximum shift in retention time is about half of the chromatographic peak width, which indicates that all molecular species have overlapping elution profiles (Figure 1C). We expect that this degree of shifting typically does not alter isotope ratios, as long as a moderate amount of labeling is used and the mass spectrum is integrated over a sufficiently large retention time window.

The quantification of $\mathrm{D}$ in intact lipids is complicated by ${ }^{13} \mathrm{C}$, which is naturally present in lipids at about $1.1 \%$. Mass gained by ${ }^{13} \mathrm{C}$ or $\mathrm{D}$ cannot be distinguished by current TOF mass analyzers (resolving power $\sim 30,000)$. Resolving the minute mass difference $(\sim 3 \mathrm{mDa})$ is possible 
for small lipids $(<600 \mathrm{Da})$ by Orbitrap MS, but this approach is currently not ideal for LC-MS due to the long scan times $(\sim 1$ second). So we need a procedure to determine the gain of isotopic label indirectly by comparing lipid extracts from bacterial cultures grown with and without label. The average molecular weights of the two mass distributions are calculated and their difference, $\Delta M W$, interpreted as the mass gained by $\mathrm{D}$ incorporation (Figure 2). The fractional abundance of $\mathrm{D}$ in a lipid $\left({ }^{2} F_{\text {LIPID }}\right)$ is then calculated by dividing $\Delta M W$ by the number of $\mathrm{C}$-bound hydrogens. Using this method, glycerophospholipids produced by $E$. coli grown in $4 \% \mathrm{D}_{2} \mathrm{O}$ yield a ${ }^{2} F_{\text {LIPID }}$ of about $2.5 \%$. Values lower than $4 \%$ are expected because hydrogen atoms from the unlabeled carbon source are incorporated into the lipids and biosynthetic enzymes favor ${ }^{1} \mathrm{H}$ over $\mathrm{D}$ due to kinetic isotope effects. Note that this calculation assumes that $\mathrm{N}$ - and $\mathrm{O}$-bound hydrogen atoms equilibrate fully with water during extraction and chromatography ${ }^{[31]}$. Additionally, the natural level of D, which is about $0.015 \%$, is neglected for the purpose of this study.

In order to evaluate the utility of $\mathrm{D}_{2} \mathrm{O}$-labeling lipidomics for estimating bacterial growth rates, we grew E. coli cultures in glucose minimal medium ranging from $0.0156 \%$ (natural 130 abundance) to $4 \%{ }^{2} F_{\text {WATER }}$ and quantified the glycerophospholipids PE and PG. ${ }^{2} F_{\text {LIPID }}$ increases linearly $\left(\mathrm{R}^{2}>0.99\right)$ with ${ }^{2} F_{\text {WATER }}$ (Figure $3 \mathrm{~A}$ and $\left.\mathrm{B}\right)$. Analyzing the same samples on a $\mathrm{Q}$ Exactive Plus Orbitrap operated at $\mathrm{R}=35,000$, yields nearly identical slopes. Assuming a detection limit of $0.03 \%{ }^{2} F_{\text {LIPID }}$ for $\mathrm{D}_{2} \mathrm{O}$-labeling lipidomics, we suggest that incubating cells for 15 minutes with $5 \%$ ${ }^{2} F_{\text {WATER }}$ is a useful range to quantify lipid biosynthesis from microbes growing at one doubling 135 per day (Figure 3C). These boundary conditions indicate that $\mathrm{D}_{2} \mathrm{O}$-labeling lipidomics can be developed further into a method to estimate microbial growth rates in environmental samples ${ }^{[3]}$. An important consideration for microcosm incubations is that two separate populations of molecules co-occur after labeling, a pool that contains low natural D abundance and a new pool that is enriched in D. High labeling strength would create molecules that occur further away from the monoisotopic mass in the spectrum and become difficult to quantify. 
Ionization conditions can affect isotopologue distributions and thus alter isotope ratios ${ }^{[32]}$. We varied the injected sample amount, ionization mode, capillary voltage, desolvation temperature and desolvation gas flow without noticing significant changes. For instance, PE(16:0/16:1) had a ${ }^{2} F_{\text {LIPID }}$ of $2.549 \pm 0.003(1 \sigma)$ in positive ionization mode and $2.567 \pm 0.019$ in negative mode (lower precision due to increased background noise). Less abundant analytes have greater standard deviations. PE(16:0/16:0), which was 10-times less abundant, had a ${ }^{2} F_{\text {LIPID }}$ of $2.432 \pm 0.078$ (positive mode). These trials show that the D abundance of lipids can be measured reproducibly. However, the most intense signals must be within the linear range of the mass analyzer and the detector must not be in dead time on a TOF instrument. Also, isotopologue patterns that are affected by co-eluting compounds have to be excluded. In our UPLC setup this was the case for some cardiolipins $(\mathrm{m} / \mathrm{z}>$ $1,200)$.

For the calculation of ${ }^{2} F_{\text {LIPID }}$ we assume that C-bound hydrogens do not exchange with solvent water during sample preparation and electrospray ionization, while $\mathrm{N}$ - and O-bound hydrogens fully equilibrate. If this is not the case, we would obtain inaccurate ${ }^{2} F_{\text {LIPID }}$ values ${ }^{[33,34]}$. To test for H/D exchange we compare UPLC-ESI-TOF with GC/P/IRMS, which quantifies near-natural isotopic composition of fatty acids ${ }^{[35]}$. Albeit the two methods are distinct in many ways, they should yield a similar linear relationship between ${ }^{2} F_{\text {WATER }}$ and ${ }^{2} F_{\text {LIPID }}{ }^{[36,37]}$. For lipidomics, we determine an average slope for intact lipids produced by E. coli in glucose minimal medium of $0.577 \pm 0.003$ (Figure S1; ${ }^{2} F_{\text {WATER }}$ between 0.125 and $4 \%$ ). Slightly higher slopes have

160 been reported previously for $E$. coli fatty acids using GC/P/IRMS (0.65 \pm 0.04 for C16:0, 0.60 \pm 0.02 for $\mathrm{C} 16: 1$ and $0.63 \pm 0.03$ for $\mathrm{C} 18: 1)^{[37]}$. Growth on acetate raises ${ }^{2} F$ in $E$. coli fatty acids analyzed by GC/P/IRMS, and it does so also for intact lipids measured by LC-MS (Figure S1). Overall, we obtain comparable slopes by lipidomics and GC/P/IRMS. 
In order to further constrain H/D exchange, we dissolved $\mathrm{PE}(18: 0 / 18: 1)$ in acetonitrile, added $\mathrm{H}_{2} \mathrm{O}$ or $\mathrm{D}_{2} \mathrm{O}$ (sold as 99.9 at $\%$ D), and recorded mass spectra by direct infusion. The addition of $\mathrm{D}_{2} \mathrm{O}$ shifts the mass spectrum by four units in positive ionization mode, as expected for an analyte that has four non $\mathrm{C}$-bound hydrogens (Figure 4). The $\Delta M W$ of 3.86 suggests that the four exchangeable hydrogens in $\mathrm{PE}(18: 0 / 18: 1)[\mathrm{M}+\mathrm{H}]+$ have an average probability of $97.4 \%$ to contain D. A theoretical spectrum that assumes four positions in the ion to have this probability for $\mathrm{D}$ closely matches the measured spectrum. Importantly, $\mathrm{D}_{2} \mathrm{O}$ addition does not yield detectable signal beyond a shift of the unlabeled distribution by four mass units. Such isotopologues would occur if the exchange of $\mathrm{C}$-bound hydrogens occurs at high rates during ESI. Absence of these signals indicates that C-bound hydrogens exchanged at least 2000-fold slower than non-C-bound hydrogens, which is in line with prior assessments of C-bound hydrogen exchange ${ }^{[38]}$. Together these tests imply that for lipids labeled well above natural D-abundance, no relevant artifacts of ${ }^{2} F_{\text {LIPID }}$ values due to exchange of C-bound hydrogen are likely in UPLC-ESI-TOF.

With the addition of small quantities of $\mathrm{D}_{2} \mathrm{O}$ to pure cultures we have an opportunity to measure lipid isotope ratios and absolute concentrations simultaneously and compare the two quantifications side by side. Our test case here is the lipid metabolism of E. coli during the transition from stationary phase into exponential growth. Cells from two stationary phase precultures ( $u$ :'unlabeled' and $l:$ 'labeled' in $4 \%{ }^{2} F_{\text {WATER }}$ ) were used to inoculate two cultures each of unlabeled $(U)$ or labeled $(L)$ medium (Figure 5). Four growth cultures $(u U, l U, u L, l L)$ were sampled to determine optical density $\left(\mathrm{OD}_{600}\right)$, as well as bulk protein and lipid concentrations. During the 160 minutes of labeling, cells divided three to four times (Figure S2).

In these tests, the stationary phase $E$. coli cultures contain a high proportion of cyclopropane fatty acids (CFA). Greater than $25 \mathrm{~mol} \%$ of PE and PG phospholipids contain at least one acyl chain with cyclopropyl ring. When cells resume growth, CFA abundance decreases to about 12 
mol\% (Figure 5B and C). The formation of CFAs in E. coli is a post-synthetic modification of the unsaturated phospholipids that occurs predominantly as cultures enter the stationary phase. CFA synthase has an unusual regulation that involves enzyme instability as well as transcription of the cfa gene from two distinct promoters ${ }^{[39,40]}$. This means that, although CFA synthase is synthesized at basal levels throughout the growth curve, a transient spike in activity occurs during the log-to-stationary phase transition. In agreement with this regulation, CFAs largely dilute out during stationary-to-log phase transition. Using $\mathrm{D}_{2} \mathrm{O}$-labeling lipidomics we detect small levels of production of CFA lipids as well as D incorporation, which shows that CFA lipids were actively made during stationary phase exit (Figure 5D).

Untargeted labeling reveals striking differences between phospholipids. Here we describe the $u L$ scenario in detail. Some $\mathrm{D}_{2} \mathrm{O}$-labeling patterns fit an exponential growth model (Figure 6). Other lipids, in particular CFA-containing lipids, were inconsistent with simple exponential de novo production. For them the growth model needs to be extended. We include a parameter that accounts for lipid biomass in the inoculum that is inactive, i.e. not exponentially reproduced during the stationary-to-log phase transition (see Methods for details).

The isotopic labeling patterns of E. coli phospholipids are dominated by their two fatty acyl chains, as they contain most of the C-bound hydrogens. A major trend we notice is that lipids that contain unsaturated fatty acids label rapidly, while fully saturated lipids incorporate label more steadily (Figure 6; also Figure S3 and S4). In E. coli, unsaturated fatty acids are made during de novo fatty acid biosynthesis and not generated by modification of saturated fatty acids or phospholipids ${ }^{[29]}$. The faster labeling of unsaturated lipids we observe thus likely reflects that the unlabeled inoculum contained little unsaturated phospholipids, because most got converted into CFA during stationary phase. A second common trend is that most CFA-containing lipids show slow initial increase of ${ }^{2} F_{\text {LIPID }}$ and often do not reach full saturation levels. This reflects that CFA lipids are only produced in small quantities after inoculation and hence a large proportion of 
unlabeled material is carried over from stationary phase. As CFA formation is a post-synthetic modification, labeling of CFA lipids additionally depends on the prior labeling of the precursor pool.

Interestingly, the two common trends we observe, namely slower labeling of saturated lipids compared to unsaturated lipids and slow and incomplete labeling of CFA-containing lipids, do not apply to all phospholipids. For example, PE(16:0/18:1) and PG(16:0/18:1) have distinct labeling patterns (Figure S3). Generally, the labeling of PE lipids that have one unsaturated and one saturated straight chain fatty acyl reveal a significantly larger proportion of unlabeled lipid compared to their PG analogs. Distinct labeling dynamics also occur for some CFA lipids. While most CFA lipid pools label slowly and do not reach high labeling, production of PG(14:0/16:0(Cp)) is stimulated so that it gains label rapidly and to high levels (Figure 6). This lipid occurs only in trace amounts in stationary phase, as does its precursor PG(14:0/16:1). Therefore, the material produced during outgrowth of the cultures is highly labeled and dominates the PG(14:0/16:0(Cp)) pool. Overall, these tests indicates that $\mathrm{D}_{2} \mathrm{O}$ addition allows a readout of how much of the material has been newly synthesized even for minority components, whose absolute concentrations can be challenging to quantify in complex lipid extracts.

The results so far indicate that lipidomics can be used to measure bacterial lipid biosynthesis in pure cultures. If $\mathrm{D}_{2} \mathrm{O}$-labeling lipidomics could quantify microbial growth reliably in situ, this might for example enable the use of microcosm incubations to test how different drugs impact the microbial community of individual patients. A disease context that is well-suited to assess the practicability of $\mathrm{D}_{2} \mathrm{O}$-labeling lipidomics for complex samples are cystic fibrosis (CF). These chronic lung infections contain heterogeneous mixtures of human biomass and microorganisms. Some of the bacteria in CF lungs become pathogenic and tend to develop drug-resistant phenotypes. In previous 
work on $\mathrm{D}_{2} \mathrm{O}$-labeled expectorated $\mathrm{CF}$ sputum we have examined the growth of $S$. aureus via $\mathrm{D} / \mathrm{H}$ ratios of anteiso-fatty acids, an abundant fatty acids of this pathogen (3). It is important, however, that anteiso-fatty acids are produced also by other bacterial species. In the context of CF sputum, Prevotella melaninogenica and Stenotrophomonas maltophilia are relevant sources of anteiso-C15:0 and anteiso-C17:0 fatty acids in some CF patients ${ }^{[3,41,42]}$. Certain phospholipids, specifically those that contain anteiso fatty acyls, may therefore be more specific markers of S. aureus in CF infections and could be used to assess activity of the pathogen by lipidomics. To evaluate this hypothesis, we analyzed samples that had been collected and characterised as part of a longitudinal study of CF patients undergoing pulmonary exacerbations ${ }^{[42]}$.

A lipid that is appears well-suited to monitor the growth of S.aureus is $\mathrm{PG}(a-\mathrm{C} 15: 0 / a-\mathrm{C} 17: 0)$. This compound was detected in lipid extracts of $S$. aureus and its structure assigned based on the $m / z$ of the molecular ion in positive and negative ionization mode as well as MS/MS fragmentation spectra. Subsequently, signals from this lipid were also detected in expectorated sputum from several CF patients with S. aureus infection (Figure 7). Two patient 250 whose lung infections did not contain S. aureus showed no signal corresponding to PG(a-C15:0/a-C17:0). Based on these observations $\mathrm{PG}(a-\mathrm{C} 15: 0 / a-\mathrm{C} 17: 0)$ in CF sputum appears to be an specific marker for S. aureus in CF sputum.

Microbial lipid metabolites make up only a minute fraction of the total lipid content of CF sputum. The high sensitivity of ESI mass spectrometry allows detection of trace components, 255 however, we had to use concentrated lipid extracts to yield sufficiently high signal intensities for the target analyte. In order to minimize contamination of the mass spectrometer, we only collected MS data at retention times that are needed to detect abundant phospholipids of $S$. aureus (5-8 min.). Labeling of $\mathrm{CF}$ sputum with $4 \% \mathrm{D}_{2} \mathrm{O}$ for 1 hour resulted in $0.054 \pm 0.04$ at $\% \mathrm{D}$ enrichment of PG(a-C15:0/a-C17:0). This value can be used to estimate that the average generation time of S. aureus was approximately one cell doubling per day. This estimation is based on a previously 
established procedure that takes into account diffusion of the label, cell maintenance and other factors ${ }^{[3]}$. For comparison, the generation time estimated by the $\mathrm{D} / \mathrm{H}$ ratio of anteiso-C15:0 fatty acid in this sample was 3.3 days ${ }^{[42]}$. The slower estimate based on GC isotope-ratio MS could for example be caused by contributions of anteiso-C15:0 from other sources or variability in the production rates of anteiso-C15:0 containing phospholipids in S. aureus. In summary, these initial tests indicate that it is possible to measure the activity of microbial pathogens in situ by $\mathrm{D}_{2} \mathrm{O}$-labeling lipidomics. Its main benefits are that LC-MS has increased species specificity, requires smaller sample amounts, it is faster than alternative MS methods ${ }^{[43]}$. Furthermore, $\mathrm{D}_{2} \mathrm{O}$-lipidomics can be performed on instrumentation that is available in many biomedical laboratories.

\section{CONCLUSIONS}

The combination of $\mathrm{D}_{2} \mathrm{O}$-labeling and lipidomics allows a robust isotope ratio measurement, which reveals dynamic aspects of biosynthesis not accessible from absolute concentrations alone. The technology is also sufficiently sensitive to be adapted for environmental samples. Based on this study we encourage the development of LC-MS assays for the analysis of microbial growth in microbiome samples. Routine methods to measure bacterial growth in clinical samples are important to better understand microbial physiology in infections and improve diagnostics.

A critical parameter for $\mathrm{D}_{2} \mathrm{O}$-labeling lipidomics is labeling strength. Very high concentrations of $\mathrm{D}_{2} \mathrm{O}$ (e.g., 20 to $\sim 100 \%$ ) are tolerated by microorganisms and can be used to monitor biosynthesis ${ }^{[3,4]}$. For LC-MS high labeling strengths are not desirable because they cause broad isotopic distributions. Quantification would become especially difficult when only a small proportion of the lipid is newly produced. In such a scenario, the labeled lipid would have a broad mass distribution far from the monoisotopic mass and potentially even overlap with other compounds. Interestingly, the ratio $\mathrm{M} 1 / \mathrm{M} 0$ increases approximately linearly with ${ }^{2} F_{\text {LIPID }}$ 
285 (Figure S5). M1/M0 could be a simple readout of D incorporation in environmental samples. When we assume an excess of unlabeled over labeled lipid, as it is the case for many environmental incubations, we anticipate an optimal labeling strength that causes the greatest change of the $\mathrm{M} 1 / \mathrm{M} 0$ ratio. This is achieved when the $\triangle M W$ of the newly-made lipid is about $+1.5 \mathrm{Da}$. Overall, a concentration of 2-3\% ${ }^{2} F_{\text {WATER }}$ seems most suited for environmental microcosm incubations. The optimal value will depend on the complexity of the lipid sample, i.e. whether D incorporation can be assessed from isotopologue distributions or M1/M0 ratio. Another consideration is that the fraction of $\mathrm{D}$ that enters the lipid varies with microbial metabolism ${ }^{[37,44]}$. We estimate that the combination of $\mathrm{D}_{2} \mathrm{O}$-labeling and lipidomics as used here can roughly quantify growth rates greater than one cell doubling per day after labeling for 15 minutes. we have done here for 27 abundant glycerophospholipids in E. coli. As lipid extracts from tissues or environmental samples are much more complex, initial chemical fractionation of lipids could be used to make data analysis more tractable. Isotope ratios should be little affected by chemical separation and thus $\mathrm{D}_{2} \mathrm{O}$-labeling lipidomics can be optimized to a specific ecosystem. The readout that $\mathrm{D}_{2} \mathrm{O}$-labeling lipidomics enables can be applied to the study of microbial growth rates in clinical samples. It can, for example, also be applied to differentiate biologically active from inactive biomass, necromass, and contaminants.

Currently, differences in the lipid composition between microbes are already used to identify strains by chemotaxonomy ${ }^{[45,46]}$. By combining large-scale lipid detection with the 305 quantification of isotopic labeling, new applications might become possible. These include identifying microbial adaptations to drugs, determining instantaneous microbial growth rates and forecasting composition of microbial community composition after exposure to a stressor. Recording isotope labeling dynamics of lipids can help to rationalize microbial lipid function and metabolism. These efforts will benefit from related lines of research in environmental microbiology 
310 and in human physiology that measure the synthesis and turnover of lipids with isotope labeling lipidomics, mass isotopomer distribution analysis or biomarkers analysis ${ }^{[8,21,47]}$.

\section{MATERIALS AND METHODS}

\section{Deuterium-enriched growth medium}

M9 minimal medium was prepared with $3.8 \mu \mathrm{M}$ thiamine pyrophosphate and glucose $(22.2 \mathrm{mM})$ or

315 sodium acetate $(15 \mathrm{mM})^{[48]}$. All media were sterilized by filtration $(0.2 \mu \mathrm{m})$. D content was adjusted by isotope dilution (measured by weight) of $\mathrm{D}_{2} \mathrm{O}$ (D, 99.9 at $\%$; Cambridge Isotope Laboratories) with natural abundance water (MilliQ, EMD Millipore) of known isotopic composition. ${ }^{2} F_{\text {WATER }}$ of culture medium was measured on a DLT-100 liquid water isotope analyzer (Los Gatos Research). Samples were analyzed in three technical replicates, each comprising 10-12 injections. Samples with D abundances close to natural abundance were calibrated against standards ranging from $0.0136 \%$ to $0.0200 \%{ }^{2} F_{\text {WATER }}$ (corresponding to $\delta \mathrm{D}$ values from -124 to $+287 \%$ ). These were in turn calibrated against the VSMOW, GISP, and SLAP international standards ${ }^{[49]}$. More enriched samples were measured against working standards made in-house, ranging from $0.050 \%$ to $0.150 \%{ }^{2} F_{\text {WATER }}$. The presence of doubly-substituted species (D-O-D) was not taken into consideration due to fast equilibration of water molecules below $0.0150 \%$. Samples beyond this scale were no longer in the linear response range of the instrument, and we calculated ${ }^{2} F_{\text {WATER }}$ based on the gravimetric preparation of the medium. Their ${ }^{2} F$ values were confirmed by water isotope analysis after dilution with natural abundance water of known isotopic composition.

\section{E. coli cultures}

330 Escherichia coli K-12 (FRAG1) was streaked on LB agar plates for single colonies and used to inoculate $6 \mathrm{~mL}$ precultures of M9 minimal medium with glucose as carbon source ${ }^{[50]}$. All cultures were incubated at $37^{\circ} \mathrm{C}$ and shaking at $250 \mathrm{rpm}$ (Innova 44 shaker, New Brunswick Scientific). Cultures were checked by phase contrast microscopy (Axio Scope.A1, Zeiss). Optical density (OD) 
was measured at $600 \mathrm{~nm}$ wavelength (DU 800 spectrophotometer; Beckman Coulter).

To investigate the detection limits of ${ }^{2} F_{\text {LIPID }}$ and the comparison of LC-MS with GC/P/IRMS, precultures were grown for 20 hours at natural D abundance. $75 \mu \mathrm{L}$ were used to inoculate $150 \mathrm{~mL}$ medium in $1 \mathrm{~L}$ Erlenmeyer flasks. For analysis with GC/P/IRMS the medium had a ${ }^{2} F_{\text {WATER }}$ in the range from $0.0142 \%$ to $0.0202 \%(\delta \mathrm{D}-90$ to $+300 \%$ ). For analysis with LC-MS the medium had a D content of $0.0142 \%$ to $4 \%{ }^{2} F_{\text {WATER }}$. Cultures were harvested at $\mathrm{OD}_{600} 0.2-0.3$ by chilling $50 \mathrm{~mL}$ culture in ice and centrifugation at $4{ }^{\circ} \mathrm{C}$ for $20 \mathrm{~min}$ at 5,000 $\times \mathrm{g}$. Cell pellets were frozen in liquid nitrogen and stored at $-20^{\circ} \mathrm{C}$.

For monitoring change of ${ }^{2} F_{\text {LIPID }}$ during stationary phase exit, two precultures $(0.0142 \%$ and $4 \%$ ${ }^{2} F_{\text {WATER }}$ ) were centrifuged at $15{ }^{\circ} \mathrm{C}$ for 10 minutes at $15,000 \times \mathrm{g}$. The pellets were resuspended in prewarmed medium $\left(0.0142 \%\right.$ or $\left.4 \%{ }^{2} F_{\text {WATER }}\right)$ and used to inoculate $200 \mathrm{~mL}$ M9 glucose medium at an initial $\mathrm{OD}_{600}$ of 0.1 . This yielded four combinations $(u U, u L, l U, l L)$ of unlabeled/labeled inoculum $(u, l)$ in unlabeled/labeled growth medium $(U, L)$. Aliquots were incubated and sampled as described above $(20$ minutes: $40 \mathrm{~mL}, 50$ minutes: $30 \mathrm{~mL}, 100$ minutes: $30 \mathrm{~mL}$ and 150 minutes: 30 $\mathrm{mL}$ ). At each time point, $\mathrm{OD}_{600}$ was recorded and protein content of the bacterial culture was measured via BCA protein assay (Thermo Scientific) from a cell pellet ( $2 \mathrm{~mL}$ aliquot, centrifuged at $4{ }^{\circ} \mathrm{C}$ for 2 minutes at $16,900 \times \mathrm{g}$ ). Maximum $\mathrm{OD}_{600}$ was 0.8 for cultures $u U, u L$ and $1.2-1.3$ for cultures $l U, l L$. Before the BCA assay cells were chemically lysed (BugBuster, EMD Chemicals). Absorbance was recorded at $562 \mathrm{~nm}$ on a plate reader (Synergy 4, BioTek).

\section{Liquid chromatography mass spectrometry (LC-MS)}

For LC-MS analysis lipids were extracted based on the procedure by Matyash et al. ${ }^{[51]}$. Cell pellets were resuspended in $0.1 \%$ ammonium acetate to a protein concentration of $200 \mathrm{mg} / \mathrm{mL}(20$ $\left.\mathrm{OD}_{600} / \mathrm{mL}\right) .100 \mu \mathrm{L}$ of this suspension were added to $1.5 \mathrm{~mL}$ methanol, then $5 \mathrm{~mL}$ methyl t-butyl ether (MTBE) and a mix of standards containing PE(17:0/17:0), PG(17:0/17:0) and 14:1(3)-15:1 cardiolipin was added (Avanti Polar Lipids). After incubation in a ultrasonic bath for 1 hour, lipids 
(in top phase) were extracted by adding $1.25 \mathrm{~mL}$ water and re-extracted by addition of $2 \mathrm{~mL}$ MTBE/methanol/water (10:3:2.5). Samples were dried under $\mathrm{N}_{2}$, stored at $-20{ }^{\circ} \mathrm{C}$ and dissolved in $1 \mathrm{~mL}$ methanol/dichloromethane (9:1) for analysis by LC-MS.

LC-MS data were collected on an Acquity I-Class UPLC coupled to a Xevo G2-S TOF mass spectrometer (Waters). Intact polar lipids were separated on an Acquity UPLC CSH C18 column $(2.1 \mathrm{~mm} \times 100 \mathrm{~mm}, 1.7 \mu \mathrm{m}$; Waters $)$ at $55^{\circ} \mathrm{C}$ following a protocol established by Waters

365 Corporation and adapted in our laboratory ${ }^{[28]}$. Samples were run in three randomized instrument replicates (injection volume $5 \mu \mathrm{L}$ ). LC-TOF-MS ${ }^{\mathrm{E}}$ data was collected in positive and negative mode using electrospray ionization (ESI) with a desolvation temperature of $550{ }^{\circ} \mathrm{C}$ and source temperature of $120^{\circ} \mathrm{C}$.

Lipids were identified by the mass to charge ratio $(\mathrm{m} / \mathrm{z})$ of their molecular ion, their 370 fragmentation products in positive and negative mode, and comparison to representative standards. Note that that the assignment of $s n-1$ and $s n-2$ fatty acyl positions is tentative ${ }^{[30]}$. Lipids are named based on LIPIDMAPS classification ${ }^{[52]}$. Table S1 provides a summary of ions and retention times used for quantification. The monoisotopic intensity alone yields incorrect values for absolute lipid concentrations in labeling experiments, where some of the signal has shifted to higher masses. So we quantified the absolute concentrations of labeled lipids by comparing the sum of all intensities of its isotopologues with those of an internal standard (Figure S6). Peaks in extracted ion chromatograms were integrated using the software MAVEN ${ }^{[53]}$. Subsequent analysis was done in R ${ }^{[54]}$. Models of isotopic distribution patterns were calculated using the $\mathrm{R}$ package Isopat (Martin Loos, EAWAG, Switzerland).

In order to calculate the generation time we fit our data to an exponential growth model. We consider that the number of lipids increases linearly with the number of cells. The number of lipid molecules at time $\mathrm{t}$ are given by 


$$
N(t)=N_{a} * 2^{(t / G T)}+N_{i}
$$

where $\mathrm{N}_{\mathrm{a}}$ is the initial number of exponentially reproduced molecules, GT is the generation time and $\mathrm{N}_{\mathrm{i}}$ the number of 'inactive' molecules at time 0 . Time course data of the fractional abundance of $\mathrm{D}$ in the lipids $\left({ }^{2} F_{\text {LIPID }}\right)$ was fitted using the equation

$$
{ }^{2} F_{L I P I D}=\max *\left[\left(2^{t / G T}-1\right) /\left(2^{t / G T}+N_{i} / N_{a}\right)\right]
$$

where max is the saturation level of labeling.

For direct infusions a stock solution of PE(18:0/18:1) in chloroform (10 g/L; Avanti Polar Lipids)

was diluted to $1 \mu \mathrm{mol} / \mathrm{L}$ in $4: 1$ acetonitrile/water containing $10 \mathrm{mM}$ ammonium formate and $0.1 \%$ formic acid. $\mathrm{H}_{2} \mathrm{O}\left(0.015\right.$ at $\%$ D) or $\mathrm{D}_{2} \mathrm{O}(99.9$ at $\%$ D) was used for the water portion. $\mathrm{PE}(18: 0 / 18: 1)$ was initially dissolved in acetonitrile, later ammonium formate dissolved in water, and $0.1 \%$ formic acid was added. Direct infusion data was collected for 4 minutes at a flow rate of $0.4 \mathrm{~mL} / \mathrm{min}$. ESI and detector settings were as in LC-MS lipidomics experiments. First the $\mathrm{H}_{2} \mathrm{O}$ sample was measured, followed by washing the pumps and measuring the $\mathrm{D}_{2} \mathrm{O}$ sample.

Orbitrap measurements were performed at Thermo Fisher Scientific (San Jose, CA) using a Q Exactive Plus mass spectrometer operating at a full MS scan mode at a resolution of 35,000 (FWHM at $\mathrm{m} / \mathrm{z} 200$ ). The $\mathrm{m} / \mathrm{z}$ range was set to $\mathrm{m} / \mathrm{z} 150$ to 2000 for negative ion mode and $\mathrm{m} / \mathrm{z} 150$ to 1200 for positive ion mode. The automatic gain control target value was set at $10^{6}$ and the

400 maximum injection time was set at $50 \mathrm{~ms}$. Chromatography was performed on a Vanquish UHPLC (Thermo Fisher Scientific), but otherwise unchanged.

\section{CF sputum and $S$. aureus}

Sputum collection was approved by the Institutional Review Board at Children's Hospital Los Angeles (IRB\# CCI-13-00211). All patients were recruited from Children's Hospital Los Angeles and informed consent or assent was obtained from all study participants or from a parent or legal guardian. Information about microbial community composition and fatty acid analysis of sputum 
samples is published elsewhere ${ }^{[42]}$. Samples used for LC-MS were from Patients 1 (2nd hospitalization, day 5; method development), Patient 2 (day 2; growth rates). S. aureus negative controls: Patient 5 (day 1), Patient 7 (day 18 and 19).

Lipid extracts from S. aureus (grown in LB medium) were prepared and analyzed by LC-MS as for E. coli. For sputum, $10 \mathrm{mg}$ of lyophilized material was extracted by the same method. The lipid extract was dissolved in $100 \mu \mathrm{L}$ methanol and $0.2 \mu \mathrm{L}$ were injected for routine lipidomic profiling. Detection of anteiso-containing phospholipids in sputum was performed by injecting $5 \mu \mathrm{L}$ lipid extract and restricting flow into the ESI source to a retention time window of

$415 \quad 5-8 \mathrm{~min}$.

\section{Calculation of deuterium content in lipids $\left({ }^{2} F_{\text {LIPID }}\right)$}

The mass spectra of a labeled and unlabeled lipid were compared to determine the fractional abundance of D $\left({ }^{2} F_{\text {LIPID }}\right)$. We calculated the molecular weights of the two isotopologue distributions and dividing their difference by the number of C-bound hydrogen atoms. Note that we did not calculate the molecular weights using accurate masses. At 30,000 mass resolving power each signal M1, M2, etc contains isotopologues which differ slightly in mass. Measured masses have additional experimental uncertainties. For simplicity, we rather used the fact that each isotopologue must have gained a certain number of neutrons, for example M8 has gained a total of 8 neutrons from ${ }^{13} \mathrm{C},{ }^{2} \mathrm{H}$ etc. We used the isotopologue distribution to calculate by how many neutrons the distribution had

425 shifted with respect to the monoisotopic mass M0. This approach eliminates inaccuracies. It also avoids the complicating fact that the mass difference between a $\mathrm{D}$ and ${ }^{1} \mathrm{H}$ is not exactly 1 .

\section{GC pyrolysis isotope-ratio mass spectrometry}

$20 \mathrm{mg}$ of frozen and lyophilized cell pellet was transesterified and extracted in hexane/anhydrous methanol/acetyl chloride at $100{ }^{\circ} \mathrm{C}$ for 10 minutes ${ }^{[55]}$. The extract was concentrated under $\mathrm{N}_{2}$. Fatty 430 acid methyl esters (FAMEs) were first analyzed by gas chromatography mass spectrometry 
(GC-MS) on a Thermo-Scientific Trace / DSQ with a ZB-5ms column $(30 \mathrm{~m} \times 0.25 \mathrm{~mm}$, film thickness $0.25 \mu \mathrm{m}$ ) and PTV injector operated in splitless mode. Peaks were identified by comparison of mass spectra and retention times to authentic standards and library data.

The $\delta \mathrm{D}$ of FAMEs was measured by gas chromatography pyrolysis isotope-ratio mass spectrometry (GC/P/IRMS) on a Thermo-Scientific DELTA ${ }^{\text {plus } X P}$ with methane of known isotopic composition as the calibration standard ${ }^{[37]}$. Chromatographic conditions were identical as for regular GC-MS, and peaks were identified by retention order and relative height. Samples were analyzed in triplicate. All data were corrected for methyl $\mathrm{H}$ originating from methanol by analyzing the dimethyl derivative of a phthalic acid standard, for which the $\delta \mathrm{D}$ value of ring $\mathrm{H}$ is known. For comparison with LC-MS, $\delta$ D values were converted into fractional abundances $\left({ }^{2} F\right)$.

\section{ACKNOWLEDGEMENTS}

We thank all reviewers for comments. We are grateful to Drs. Fenfang Wu, Reto Wijker and Jesse Allen for technical advice on the use of instrumentation, and to Dr. Ajay Kasai for CF sputum collection. LC-MS data was collected at the Caltech Environmental Analysis Center (Pasadena) and with assistance of Dr. Anastasia Kalli at Thermo Fisher Scientific (San Jose, CA). This work was made possible in parts by grants from NASA (NNX12AD93G), the National Science Foundation (1224158), the National Institutes of Health (R01HL117328). IRB was funded by CEMI (Caltech) and by the Leverhulme Trust.

\section{REFERENCES}

[1] T. Korem, D. Zeevi, J. Suez, A. Weinberger, T. Avnit-Sagi, M. Pompan-Lotan, E. Matot, G. Jona, A. Harmelin, N. Cohen, A. Sirota-Madi, C. A. Thaiss, M. Pevsner-Fischer, R. Sorek, et al. Growth dynamics of gut microbiota in health and disease inferred from single metagenomic samples. Science 2015, 349, 1101.

[2] C. T. Brown, M. R. Olm, B. C. Thomas, J. F. Banfield. Measurement of bacterial replication rates in microbial communities. Nat. Biotechnol. 2016, 34, 1256. 
[3] S. H. Kopf, A. L. Sessions, E. S. Cowley, C. Reyes, L. Van Sambeek, Y. Hu, V. J. Orphan, R. Kato, D. K. Newman. Trace incorporation of heavy water reveals slow and heterogeneous pathogen growth rates in cystic fibrosis sputum. Proc. Natl. Acad. Sci. U. S. A. 2016, 113, E110.

[4] D. Berry, E. Mader, T. K. Lee, D. Woebken, Y. Wang, D. Zhu, M. Palatinszky, A.

Schintlmeister, M. C. Schmid, B. T. Hanson, N. Shterzer, I. Mizrahi, I. Rauch, T. Decker, et al. Tracking heavy water $\left(\mathrm{D}_{2} \mathrm{O}\right)$ incorporation for identifying and sorting active microbial cells. Proc. Natl. Acad. Sci. U. S. A. 2015, 112, E194.

[5] D. C. White, J. O. Stair, D. B. Ringelberg. Quantitative comparisons of in situ microbial biodiversity by signature biomarker analysis. J. Ind. Microbiol. Biotechnol. 1996, 17, 185.

[6] Å. Frostegård, A. Tunlid, E. Bååth. Use and misuse of PLFA measurements in soils. Soil Biol. Biochem. 2011, 43, 1621.

[7] D. K. Newman, C. Neubauer, J. N. Ricci, C.-H. Wu, A. Pearson. Cellular and Molecular Biological Approaches to Interpreting Ancient Biomarkers. Annu. Rev. Earth Planet. Sci. 2016, 44, 493.

[8] C. A. Lytle, M. E. Fuller, Y. M. Gan, A. Peacock, M. F. DeFlaun, T. C. Onstott, D. C. White. Utility of high performance liquid chromatography/electrospray/mass spectrometry of polar lipids in specifically per- ${ }^{13} \mathrm{C}$ labeled Gram-negative bacteria DA001 as a tracer for acceleration of bioremediation in the subsurface. J. Microbiol. Methods 2001, 44, 271.

[9] M. R. Wenk. Lipidomics: New tools and applications. Cell 2010, 143, 888.

[10] L. Wörmer, J. S. Lipp, J. M. Schröder, K.-U. Hinrichs. Application of two new LC-ESI-MS methods for improved detection of intact polar lipids (IPLs) in environmental samples. Org. Geochem. 2013, 59, 10.

[11] A. Pearson, 12.11 - Lipidomics for geochemistry, in Treatise on Geochemistry (Second Edition), (Eds: H.D. Holland, K.K. Turekian). Elsevier, Oxford, 2014, pp. 291-336.

[12] P. J. Taylor. Matrix effects: The Achilles heel of quantitative high-performance liquid chromatography-electrospray-tandem mass spectrometry. Clin. Biochem. 2005, 38, 328.

[13] D. Remane, D. K. Wissenbach, M. R. Meyer, H. H. Maurer. Systematic investigation of ion suppression and enhancement effects of fourteen stable-isotope-labeled internal standards by their native analogues using atmospheric-pressure chemical ionization and electrospray ionization and the relevance for multi-analyte liquid chromatographic/mass spectrometric procedures. Rapid Commun. Mass Spectrom. 2010, 24, 859.

[14] K. Yang, X. Han. Accurate quantification of lipid species by electrospray ionization mass spectrometry - Meet a key challenge in lipidomics. Metabolites 2011, 1, 21.

[15] H. H. Maurer. What is the future of (ultra) high performance liquid chromatography coupled to low and high resolution mass spectrometry for toxicological drug screening? J. Chromatogr. A 2013, 1292, 19.

[16] N. Zamboni, A. Saghatelian, G. J. Patti. Defining the metabolome: Size, flux, and regulation. Mol. Cell 2015, 58, 699.

[17] E. Ciccimaro, I. A. Blair. Stable-isotope dilution LC-MS for quantitative biomarker analysis. Bioanalysis 2010, 2, 311.

[18] T. Skotland, K. Ekroos, S. Kavaliauskiene, J. Bergan, D. Kauhanen, T. Lintonen, K. Sandvig. Determining the Turnover of Glycosphingolipid Species by Stable-Isotope Tracer Lipidomics. J. Mol. Biol. 2016, 428, 4856.

[19] K. Ekroos, I. V. Chernushevich, K. Simons, A. Shevchenko. Quantitative profiling of phospholipids by multiple precursor ion scanning on a hybrid quadrupole time-of-flight mass spectrometer. Anal. Chem. 2002, 74, 941.

[20] W. N. Lee, S. Bassilian, H. O. Ajie, D. A. Schoeller, J. Edmond, E. A. Bergner, L. O. Byerley. In vivo measurement of fatty acids and cholesterol synthesis using $\mathrm{D}_{2} \mathrm{O}$ and mass isotopomer analysis. Am. J. Physiol. 1994, 266, E699. 
[21] M. K. Hellerstein, R. A. Neese. Mass isotopomer distribution analysis at eight years: Theoretical, analytic, and experimental considerations. Am. J. Physiol. 1999, 276, E1146.

[22] A. D. Postle, D. C. Wilton, A. N. Hunt, G. S. Attard. Probing phospholipid dynamics by electrospray ionisation mass spectrometry. Prog. Lipid Res. 2007, 46, 200.

[23] J. Castro-Perez, S. F. Previs, D. G. McLaren, V. Shah, K. Herath, G. Bhat, D. G. Johns, S.-P. Wang, L. Mitnaul, K. Jensen, R. Vreeken, T. Hankemeier, T. P. Roddy, B. K. Hubbard. In vivo $\mathrm{D}_{2} \mathrm{O}$ labeling to quantify static and dynamic changes in cholesterol and cholesterol esters by high resolution LC/MS. J. Lipid Res. 2011, 52, 159.

[24] C. R. Fischer, B. P. Bowen, C. Pan, T. R. Northen, J. F. Banfield. Stable-isotope probing reveals that hydrogen isotope fractionation in proteins and lipids in a microbial community are different and species-specific. ACS Chem. Biol. 2013, 8, 1755.

[25] S. F. Previs, D. G. McLaren, S.-P. Wang, S. J. Stout, H. Zhou, K. Herath, V. Shah, P. L. Miller, L. Wilsie, J. Castro-Perez, D. G. Johns, M. A. Cleary, T. P. Roddy. New methodologies for studying lipid synthesis and turnover: looking backwards to enable moving forwards. Biochim. Biophys. Acta 2014, 1842, 402.

[26] J. A. McCloskey, A. M. Lawson, F. A. J. M. Leemans. Gas-chromatographic and mass-spectrometric properties of perdeuterated fatty acid methyl esters. Chem. Commun. 1967, 285.

[27] S. Wang, M. Cyronak, E. Yang. Does a stable isotopically labeled internal standard always correct analyte response? A matrix effect study on a LC/MS/MS method for the determination of carvedilol enantiomers in human plasma. J. Pharm. Biomed. Anal. 2007, 43, 701.

[28] C. Neubauer, N. F. Dalleska, E. S. Cowley, N. J. Shikuma, C.-H. Wu, A. L. Sessions, D. K. Newman. Lipid remodeling in Rhodopseudomonas palustris TIE-1 upon loss of hopanoids and hopanoid methylation. Geobiology 2015, 13, 443.

530 [29] J. E. Cronan Jr, C. O. Rock. Biosynthesis of Membrane Lipids. EcoSal Plus 2008, 3, DOI 10.1128/ecosalplus.3.6.4.

[30] D. Oursel, C. Loutelier-Bourhis, N. Orange, S. Chevalier, V. Norris, C. M. Lange. Lipid composition of membranes of Escherichia coli by liquid chromatography/tandem mass spectrometry using negative electrospray ionization. Rapid Commun. Mass Spectrom. 2007,

[31] A. Schimmelmann, A. L. Sessions, M. Mastalerz. Hydrogen isotopic (D/H) composition of organic matter during diagenesis and thermal maturation. Annu. Rev. Earth Planet. Sci. 2006, 34, 501.

[32] B. W. Patterson, R. R. Wolfe. Concentration dependence of methyl palmitate isotope ratios by electron impact ionization gas chromatography/mass spectrometry. Biol. Mass Spectrom. 1993, $22,481$.

[33] N. W. Davies, J. A. Smith, P. P. Molesworth, J. J. Ross. Hydrogen/deuterium exchange on aromatic rings during atmospheric pressure chemical ionization mass spectrometry. Rapid Commun. Mass Spectrom. 2010, 24, 1105.

[34] Y. Wang, A. L. Sessions, R. J. Nielsen, W. A. Goddard III. Equilibrium ${ }^{2} \mathrm{H} /{ }^{1} \mathrm{H}$ fractionations in organic molecules. II: Linear alkanes, alkenes, ketones, carboxylic acids, esters, alcohols and ethers. Geochim. Cosmochim. Acta 2009, 73, 7076.

[35] A. L. Sessions. Isotope-ratio detection for gas chromatography. J. Sep. Sci. 2006, 29, 1946.

[36] H. W. Kreuzer-Martin, M. J. Lott, J. R. Ehleringer, E. L. Hegg. Metabolic processes account for the majority of the intracellular water in log-phase Escherichia coli cells as revealed by hydrogen isotopes. Biochemistry 2006, 45, 13622.

[37] X. Zhang, A. L. Gillespie, A. L. Sessions. Large D/H variations in bacterial lipids reflect central metabolic pathways. Proceedings of the National Academy of Sciences 2009, 106, 12580 .

555 [38] A. L. Sessions, S. P. Sylva, R. E. Summons, J. M. Hayes. Isotopic exchange of carbon-bound 
hydrogen over geologic timescales 1. Geochim. Cosmochim. Acta 2004, 68, 1545.

[39] A.-Y. Wang, J. E. Cronan. The growth phase-dependent synthesis of cyclopropane fatty acids in Escherichia coli is the result of an RpoS (KatF)-dependent promoter plus enzyme instability. Mol. Microbiol. 1994, 11, 1009.

[40] Y. Y. Chang, J. Eichel, J. E. Cronan Jr. Metabolic instability of Escherichia coli cyclopropane fatty acid synthase is due to RpoH-dependent proteolysis. J. Bacteriol. 2000, 182, 4288.

[41] C. C. Wu, J. L. Johnson, W. E. Moore, L. V. Moore. Emended descriptions of Prevotella denticola, Prevotella loescheii, Prevotella veroralis, and Prevotella melaninogenica. Int. J. Syst. Bacteriol. 1992, 42, 536.

565 [42] C. Neubauer, A. S. Kasi, N. Grahl, D. A. Hogan, A. L. Sessions, S. H. Kopf, R. Cato, D. K. Newman. Refining the application of anteiso fatty acids as tracers of Staphylococcus aureus growth rates in cystic fibrosis sputum. To be submitted n.d.

[43] S. H. Kopf, S. E. McGlynn, A. Green-Saxena, Y. Guan, D. K. Newman, V. J. Orphan. Heavy water and ${ }^{15} \mathrm{~N}$ labelling with NanoSIMS analysis reveals growth rate-dependent metabolic heterogeneity in chemostats. Environ. Microbiol. 2015, 17, 2542.

[44] M. R. Osburn, K. S. Dawson, M. L. Fogel, A. L. Sessions. Fractionation of hydrogen isotopes by sulfate- and nitrate-reducing bacteria. Front. Microbiol. 2016, 7, 1166.

[45] N. R. Saichek, C. R. Cox, S. Kim, P. B. Harrington, N. R. Stambach, K. J. Voorhees. Strain-level Staphylococcus differentiation by $\mathrm{CeO}_{2}$-metal oxide laser ionization mass spectrometry fatty acid profiling. BMC Microbiol. 2016, 16, 72 .

[46] E. Layre, L. Sweet, S. Hong, C. A. Madigan, D. Desjardins, D. C. Young, T.-Y. Cheng, J. W. Annand, K. Kim, I. C. Shamputa, M. J. McConnell, C. A. Debono, S. M. Behar, A. J. Minnaard, et al. A comparative lipidomics platform for chemotaxonomic analysis of Mycobacterium tuberculosis. Chem. Biol. 2011, 18, 1537.

580 [47] J. Brandsma, A. P. Bailey, G. Koster, A. P. Gould, A. D. Postle. Stable isotope analysis of dynamic lipidomics. Biochim. Biophys. Acta 2017, DOI 10.1016/j.bbalip.2017.03.002.

[48] J. Sambrook, E. F. Fritsch, T. Maniatis, Others. Molecular Cloning: A Laboratory Manual. Cold Spring Harbor Laboratory Press, 1989.

[49] T. B. Coplen. New guidelines for reporting stable hydrogen, carbon, and oxygen isotope-ratio data. Geochim. Cosmochim. Acta 1996, 60, 3359.

[50] W. Epstein, M. Davies. Potassium-dependant mutants of Escherichia coli K-12. J. Bacteriol. 1970, 101, 836.

[51] V. Matyash, G. Liebisch, T. V. Kurzchalia, A. Shevchenko, D. Schwudke. Lipid extraction by methyl-tert-butyl ether for high-throughput lipidomics. J. Lipid Res. 2008, 49, 1137.

[52] E. Fahy, S. Subramaniam, R. C. Murphy, M. Nishijima, C. R. H. Raetz, T. Shimizu, F. Spener, G. van Meer, M. J. O. Wakelam, E. A. Dennis. Update of the LIPID MAPS comprehensive classification system for lipids. J. Lipid Res. 2009, 50 Suppl, S9.

[53] E. Melamud, L. Vastag, J. D. Rabinowitz. Metabolomic analysis and visualization engine for LC-MS data. Anal. Chem. 2010, 82, 9818.

[54] R Core Team. R: A language and environment for statistical computing. Vienna: R Foundation for Statistical Computing. 2016.

[55] J. Rodríguez-Ruiz, E.-H. Belarbi, J. L. G. Sánchez, D. L. Alonso. Rapid simultaneous lipid extraction and transesterification for fatty acid analyses. Biotechnol. Tech. 1998, 12, 689.

[56] S. Hiraoka, H. Matsuzaki, I. Shibuya. Active increase in cardiolipin synthesis in the stationary growth phase and its physiological significance in Escherichia coli. FEBS Lett. 1993, 336, 221. 

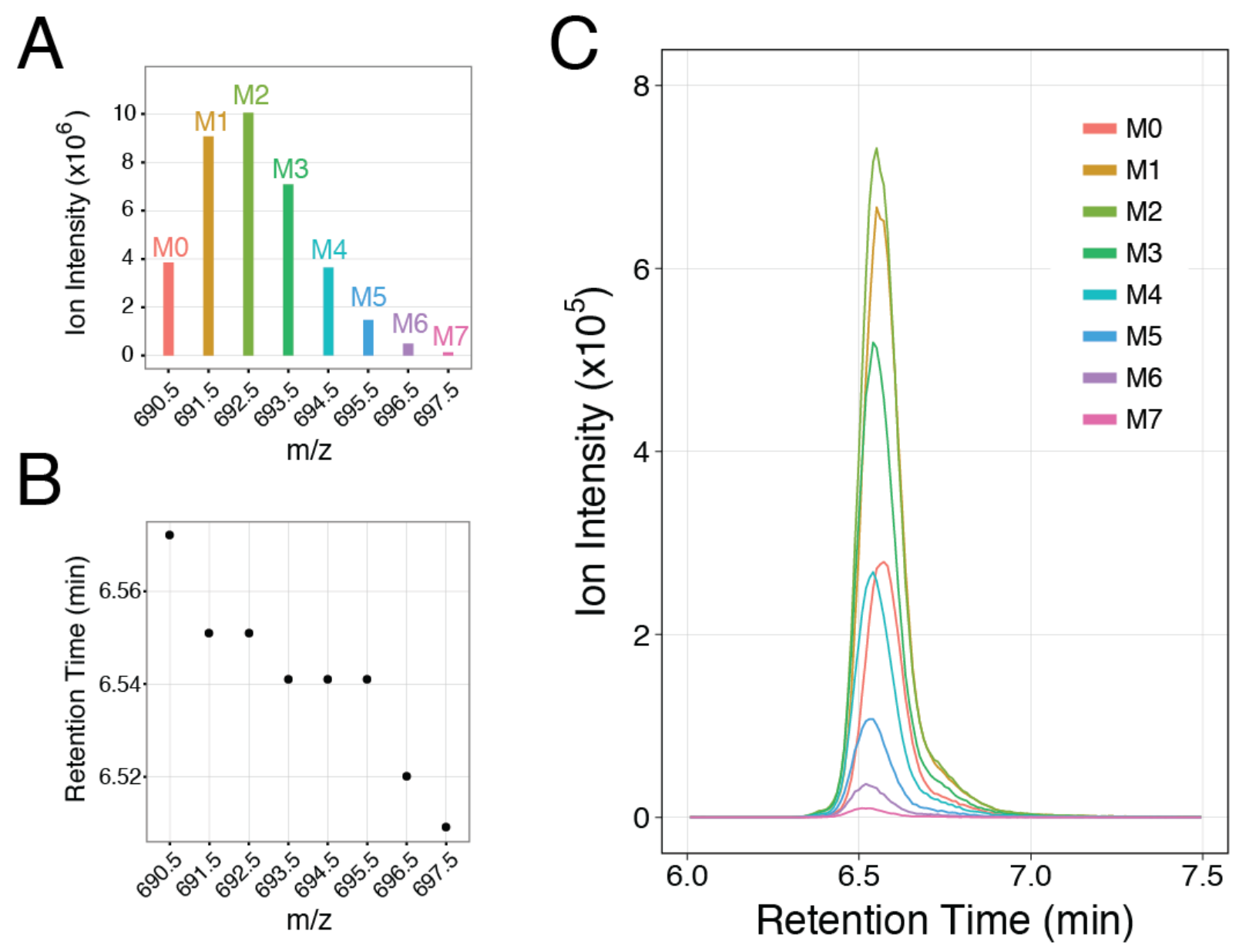

Figure 1: Deuterium-labeling shifts the chromatographic retention of lipids. LC-MS data of labeled $\mathrm{PE}(16: 0 / 16: 1)$, made by an E. coli culture grown in $4 \% \mathrm{D}_{2} \mathrm{O}$. The retention time shift was evident, yet most isotopologues co-eluted and derived isotope-ratios were not altered. (A) Isotopologue distribution (M0 to $\mathrm{M} 7 ; \mathrm{m} / \mathrm{z} \pm 0.05$ ). Data collected in positive mode. (B) Retention time at maximum ion intensity for each $\mathrm{m} / \mathrm{z}$ range. Spectra were collected at a rate of 95 scans per minute. (C) Extracted ion chromatograms for the eight $\mathrm{m} / \mathrm{z}$ ranges. 


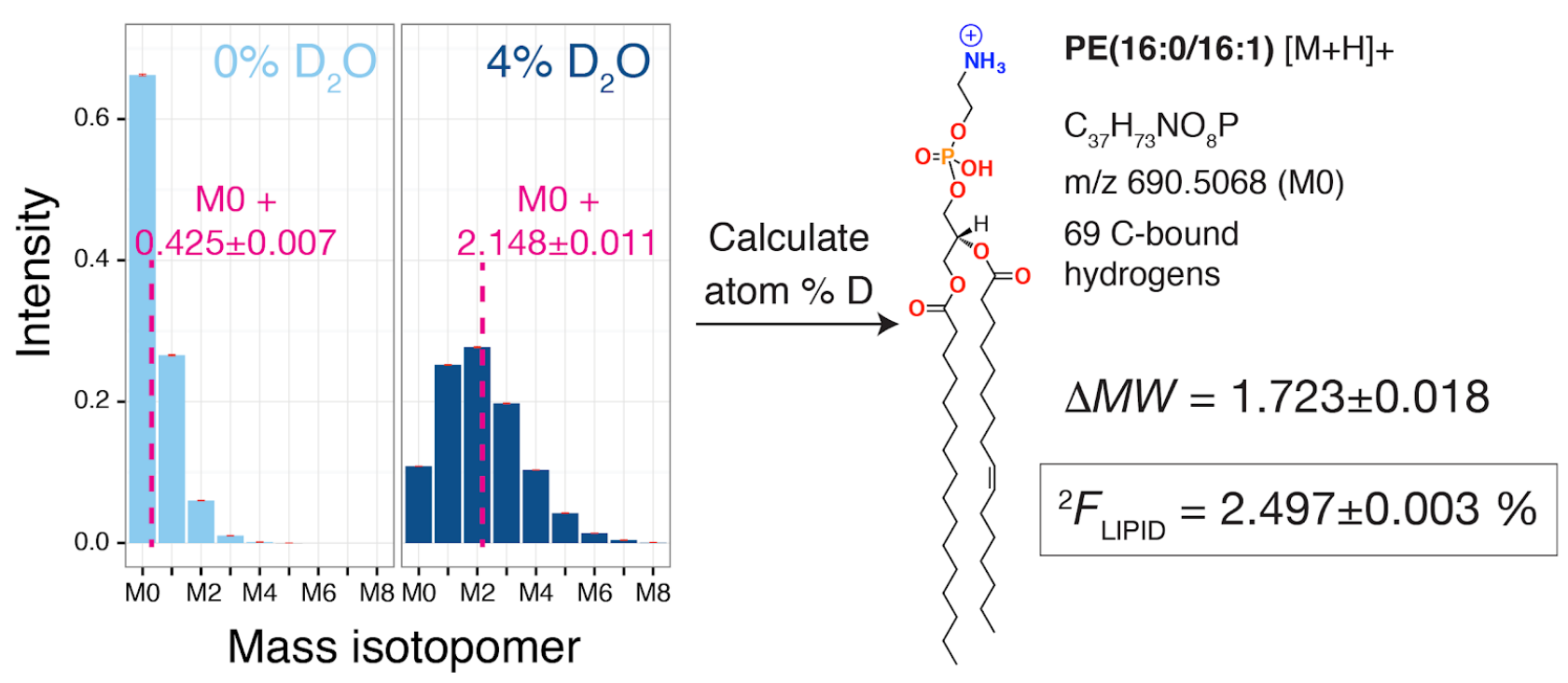

Figure 2: Calculating the deuterium content of intact lipids from an unlabeled and labeled sample. Two cultures of E. coli FRAG-1 were grown without and with $\mathrm{D}_{2} \mathrm{O}$ addition (4\%). The molecular weight was calculated from each distribution and is shown relative to M0 in magenta $( \pm 1 \sigma)$. The deuterium content of the lipid was calculated by dividing the mass difference $\Delta M W$ by

615 the number of C-bound hydrogens (for details see Methods). ${ }^{2} F_{\text {LIPID }}$ generally had a standard deviation $<0.005$ at\%. The isotopic distribution of $\mathrm{PE}(16: 0 / 16: 1)$ in positive mode is shown. Lipid extracts measured by LC-MS using injection volumes of 1,3 and $9 \mu \mathrm{L}$. Note that error bars $( \pm 1 \sigma$; magenta) are small. 

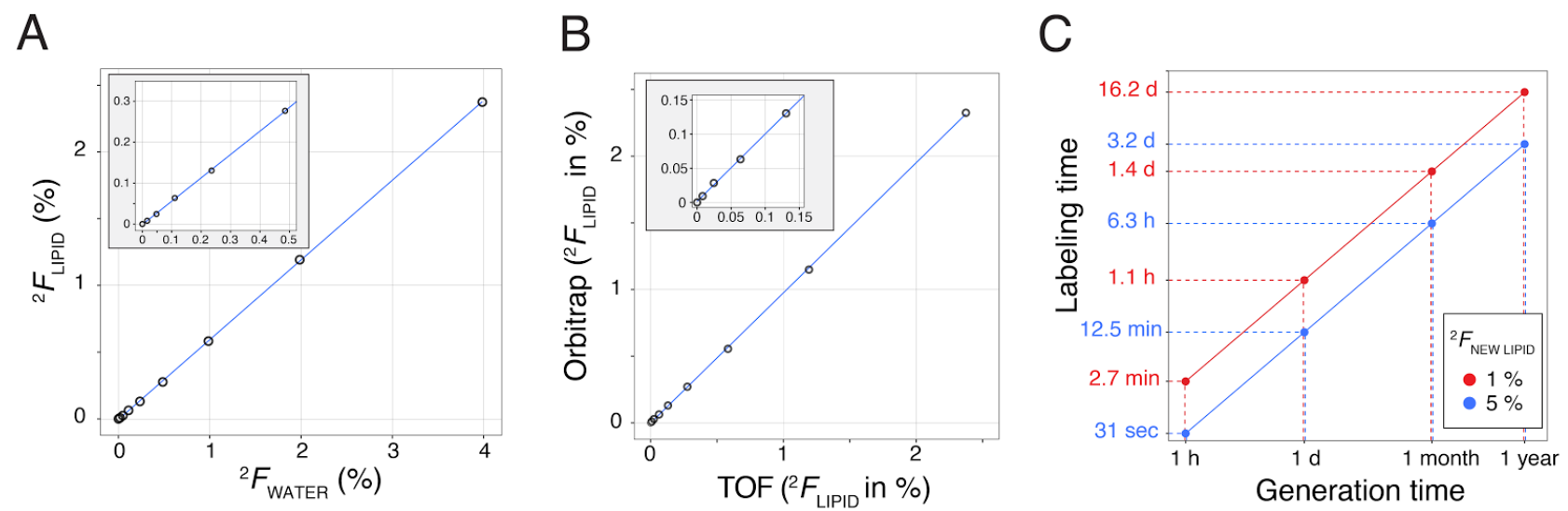

Figure 3: Isotope-ratios by lipidomics are suitable for measuring microbial growth rates in situ. (A) Overnight cultures of E. coli were grown in M9-glucose medium containing varying amounts of $\mathrm{D}_{2} \mathrm{O}$ and lipid extracts analyzed by LC-MS. The measured deuterium content of $\operatorname{PE}(16: 0 / 16: 0(\mathrm{Cp}))$ is shown. Enrichments with ${ }^{2} F_{\text {LIPID }}>0.03 \%$ were within the linear range. (B) Comparison of a Xevo G2 TOF instrument and a Orbitrap Q Exactive Plus (resolving power 35,000 at $\mathrm{m} / \mathrm{z} 200)$ using the same samples as in (A). ${ }^{2} F_{\text {LIPID }}$ values for PE(16:0/16:0(Cp)) are plotted. The linear regression has a slope of 0.976 and $\mathrm{R}^{2}$ of 0.999 . (C) Estimate of how long $\mathrm{D}_{2} \mathrm{O}$-labeling has to be performed to achieve deuterium enrichment of $+0.03 \mathrm{at} \% \mathrm{D}$ in lipids, assuming that the newly made lipid fraction $\left({ }^{2} F_{\text {NEW LIPID }}\right)$ gets labeled at $1 \%$ (red) or $5 \%$ (blue). For a microbe growing with a doubling time of one day, incubation would need to be performed for $12.5 \mathrm{~min}$ or 1.1 hours, respectively, to reach en enrichment of $0.03 \%$ over natural ${ }^{2} F_{\text {LIPID }}$. We here estimate the enrichment at time $\mathrm{t}$ using the following equation: ${ }^{2} F_{\text {LIPID }}(\mathrm{t})={ }^{2} F_{\text {NEW LIPID }} *\left(1-2^{\mathrm{t} / \mathrm{GT}}\right)$. 


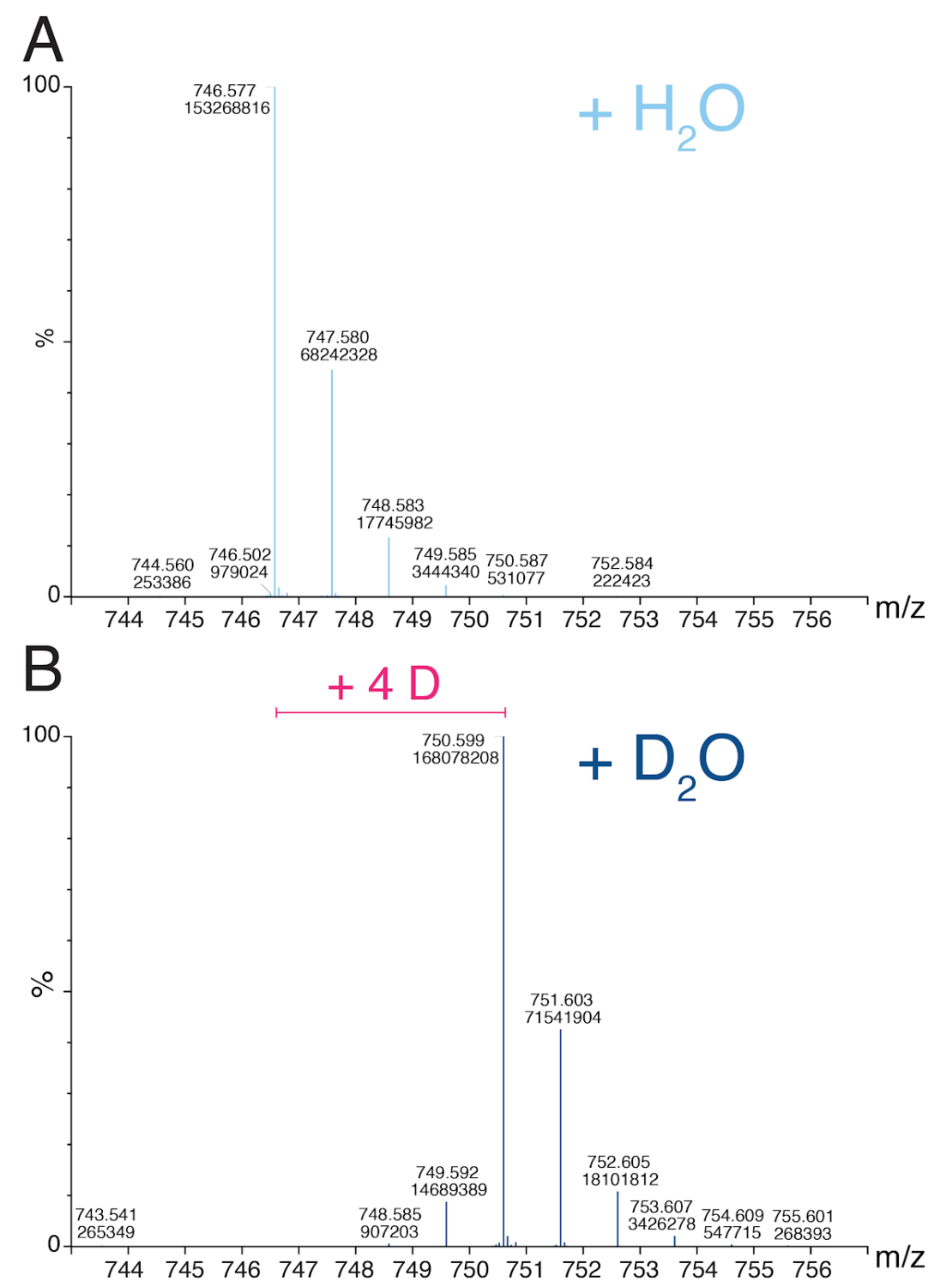

Figure 4: Direct infusion of lipid in the presence of $\mathrm{H}_{2} \mathrm{O}$ or $\mathrm{D}_{2} \mathrm{O}$ showed no exchange of C-bound hydrogens. Direct infusion mass spectra of $\mathrm{PE}(18: 0 / 18: 1)([\mathrm{M}+\mathrm{H}]+$ ion $)$ dissolved in (A) $635 \mathrm{H}_{2} \mathrm{O}\left(0.015\right.$ at $\%$ D) or $(\mathbf{B}) \mathrm{D}_{2} \mathrm{O}(99.9 \mathrm{at} \%$ D). The molecular weight shift $\Delta M W$ was 3.86 and indicated that maximally $97.4 \%$ of the four exchangeable sites exchanged to deuterium. No signal above background was detected beyond $m / z 750.6\left(\mathrm{H}_{2} \mathrm{O}\right.$ sample) and $m / z 754.6\left(\mathrm{D}_{2} \mathrm{O}\right.$ sample). The solution contained a 4:1 ratio of acetonitrile to water and was buffered with $10 \mathrm{mM}$ ammonium formate and $0.1 \%$ formic acid, which contained ${ }^{1} \mathrm{H}$. Data was acquired for 4 minutes at $0.4 \mathrm{~mL} / \mathrm{min}$ flow rate. 


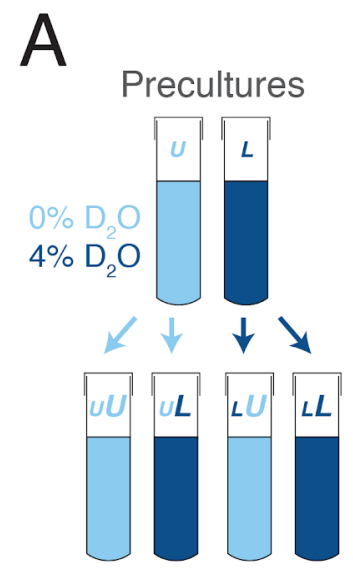

Growth curve
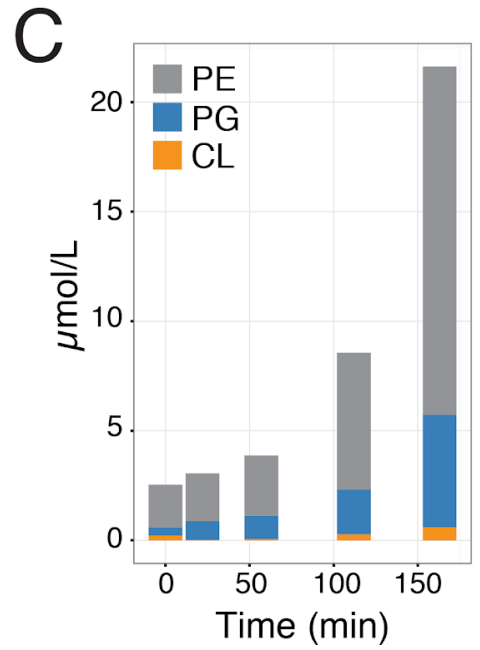

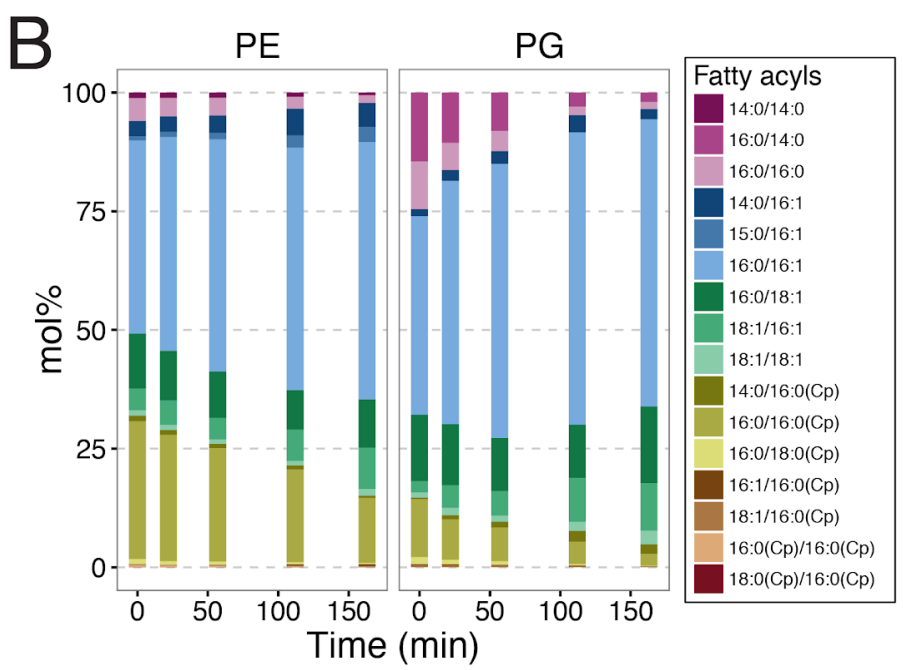

$D$

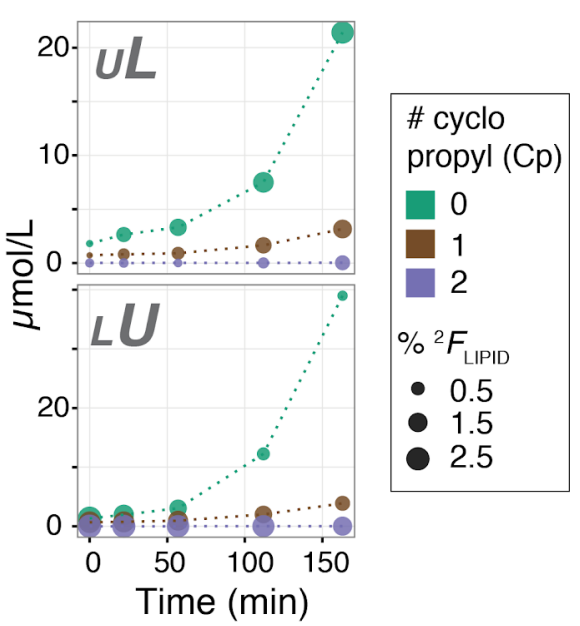

Figure 5: Lipid production in $E$. coli during stationary phase exit was followed by absolute concentrations and deuterium incorporation. (A) Four cultures were followed by lipidomics. Labeling scheme: $l=$ labeled $\left(4 \% \mathrm{D}_{2} \mathrm{O}\right.$; dark blue) stationary preculture, $u=$ unlabeled (light blue) stationary preculture, $L=$ labeled $\left(4 \% \mathrm{D}_{2} \mathrm{O}\right)$ growth culture, $U=$ unlabeled growth culture. (B) Relative abundance of PE and PG lipids. Cyclopropane fatty acyls are more abundant in stationary phase compared to growth phase (data from $u L$ culture). (C), double bonds, and cyclopropane rings (Cp) in acyl chains. (C) Quantification of lipid classes (data from $u U$ culture). Throughout the outgrowth the proportion of PE (grey) was 70-78 mol\% and PG (blue) 19-24 mol\%. CL (orange) is synthesized from two molecules of PG and often increased in stationary phase ${ }^{[56]}$. In this time course CL was $3.5 \mathrm{~mol} \%$ in the inoculum and $1.5-2.5 \mathrm{~mol} \%$ during outgrowth. (D) Time course for PE and PG lipids with 0 (green), 1 (brown) or 2 cyclopropyl rings (violet) in cultures $u L$ and $l U$. Size of the data points represents deuterium abundance in the lipid $\left({ }^{2} F_{\text {LIPID }}\right)$. 

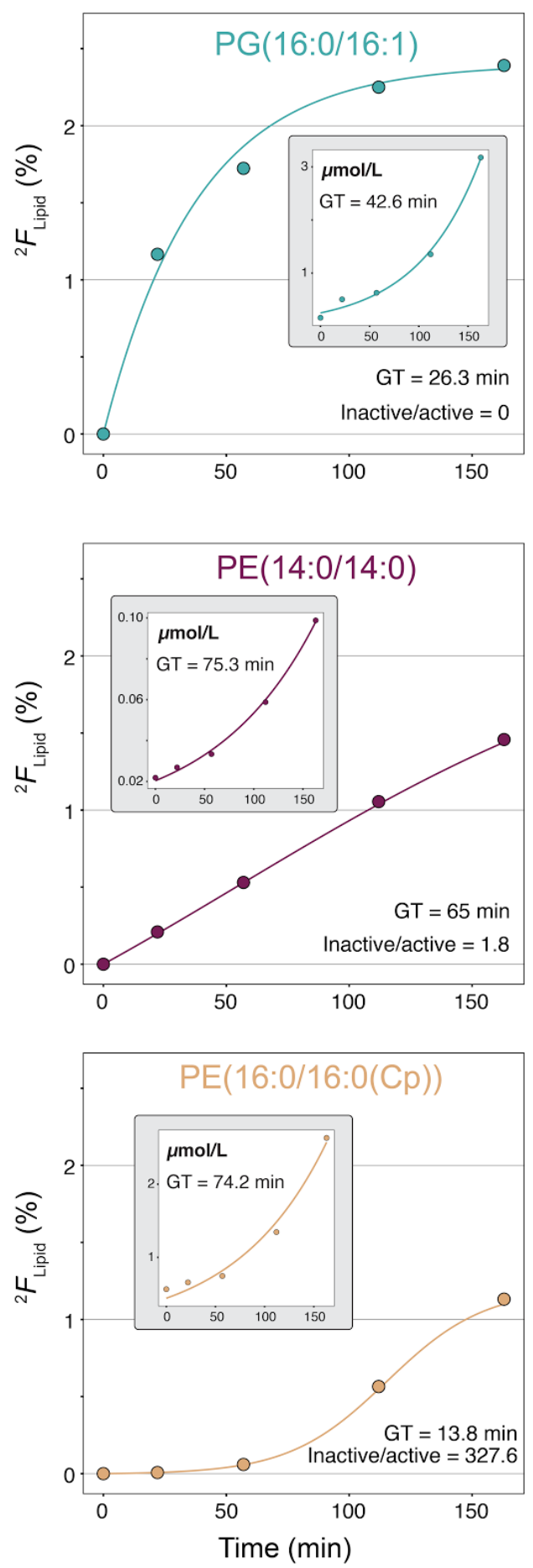

655 Figure 6: During stationary phase exit lipids have distinct labeling dynamics, which are dominated by the fatty acyl chains. The deuterium abundance $\left({ }^{2} F_{\text {LIPID }}\right)$ in three representative lipids is shown (data from culture $u L$ ). Lines represent the best fit of a growth model that estimates generation time (GT) and the ratio of inactive to active unlabeled material at time 0 (see Methods for details). The insets show absolute concentrations for comparison. See Figure S3 \& S4 for a comprehensive overview of lipids. 
A

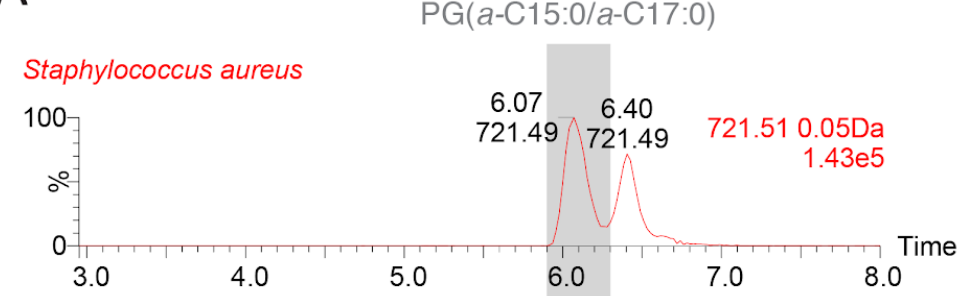

CF sputum with $S$. aureus infection

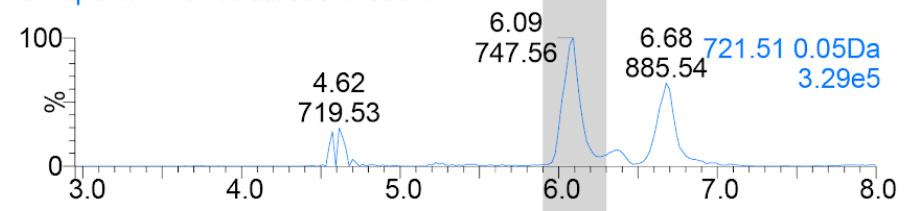

CF sputum (no S. aureus; mainly Stenotrophomonas and Prevotella)

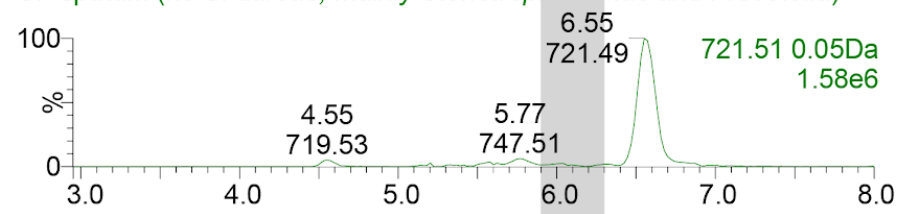

CF sputum II (no S. aureus; mainly Pseudomonas)

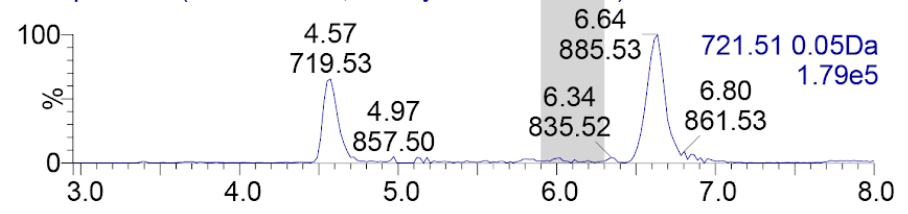

721.49

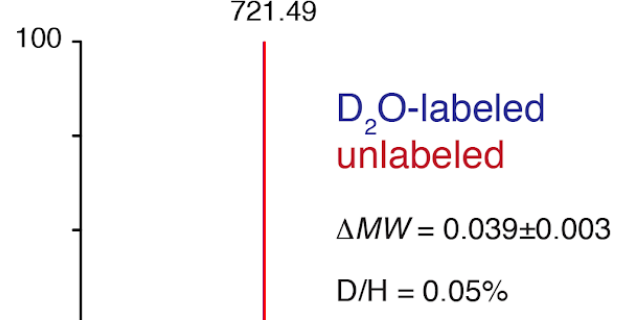

722.50

723.50

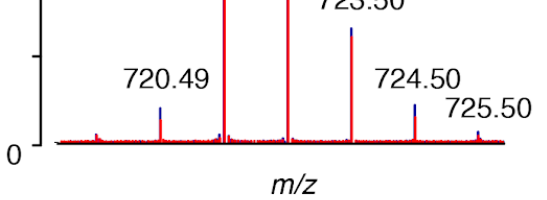

Figure 7: Growth of S. aureus in cystic fibrosis (CF) lung infections. (A) S. aureus (top) produces phospholipids that contain anteiso fatty acids. $\mathrm{PG}(a-\mathrm{C} 15: 0 / a-\mathrm{C} 17: 0)$, which has a $m / z$ 721.51 in negative ionization mode and elutes at $6.1 \mathrm{~min}$, is shown as example. In expectorated sputum from a CF patient with a $S$. aureus infection (second from top) $\mathrm{PG}(a-\mathrm{C} 15: 0 / a-\mathrm{C} 17: 0)$ is detected, while no comparable levels are detected in a controls from two patients whose infection does not contain S. aureus (bottom two panels). Note that S. aureus analytes occur only at trace abundances in sputum and concentrated lipid extracts were used to collect mass spectra. (B) Deuterium content of PG(a-C15:0/a-C17:0) from CF sputum labeled for 1 hour at $4 \% \mathrm{D}_{2} \mathrm{O}$ (blue) and unlabeled sputum (red). From the $\mathrm{D} / \mathrm{H}$ of $0.05 \%$ generation times of $S$. aureus on the order of one cell doubling per day can be inferred ${ }^{[3]}$. 


\section{SUPPLEMENTAL INFORMATION}

\section{Supplemental Figures S1-S6}

Supplemental Table S1: Information about quantified lipids. (see separate file)

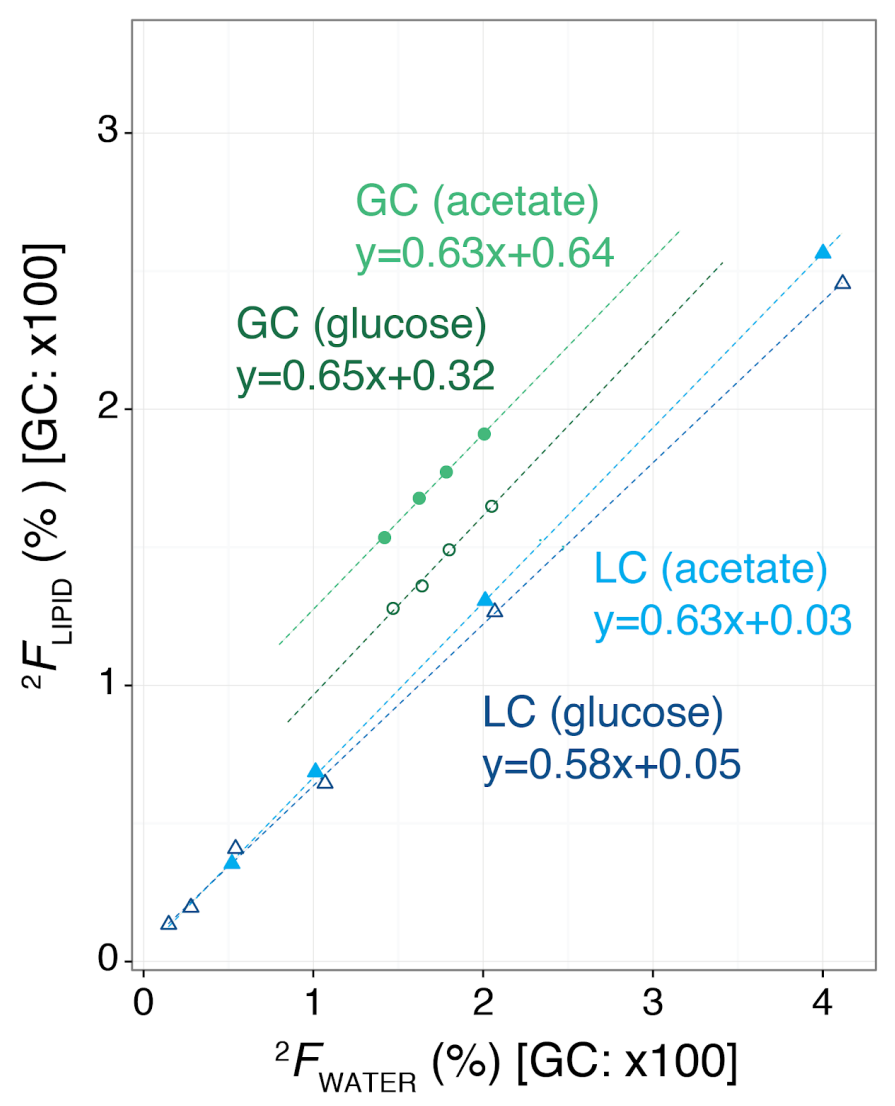
were grown on glucose (darker color) or acetate (lighter color) as carbon sources using varying amounts of deuterium in the growth medium $\left({ }^{2} F_{\text {WATER }}\right)$. For both methods deuterium content in lipids (LC) or fatty acids (GC) increases linearly with ${ }^{2} F_{\text {WATER }}$. Slopes are comparable between LC (blue triangles) and GC/P/IRMS (green circles). Data for fatty acid 16:0 (GC-MS) and PG(16:0/16:0) (LC-MS) are shown. Values for GC-MS are multiplied by a factor of 100 for easy comparison with values from LC-MS. Filled points represent glucose, empty points acetate cultures. 


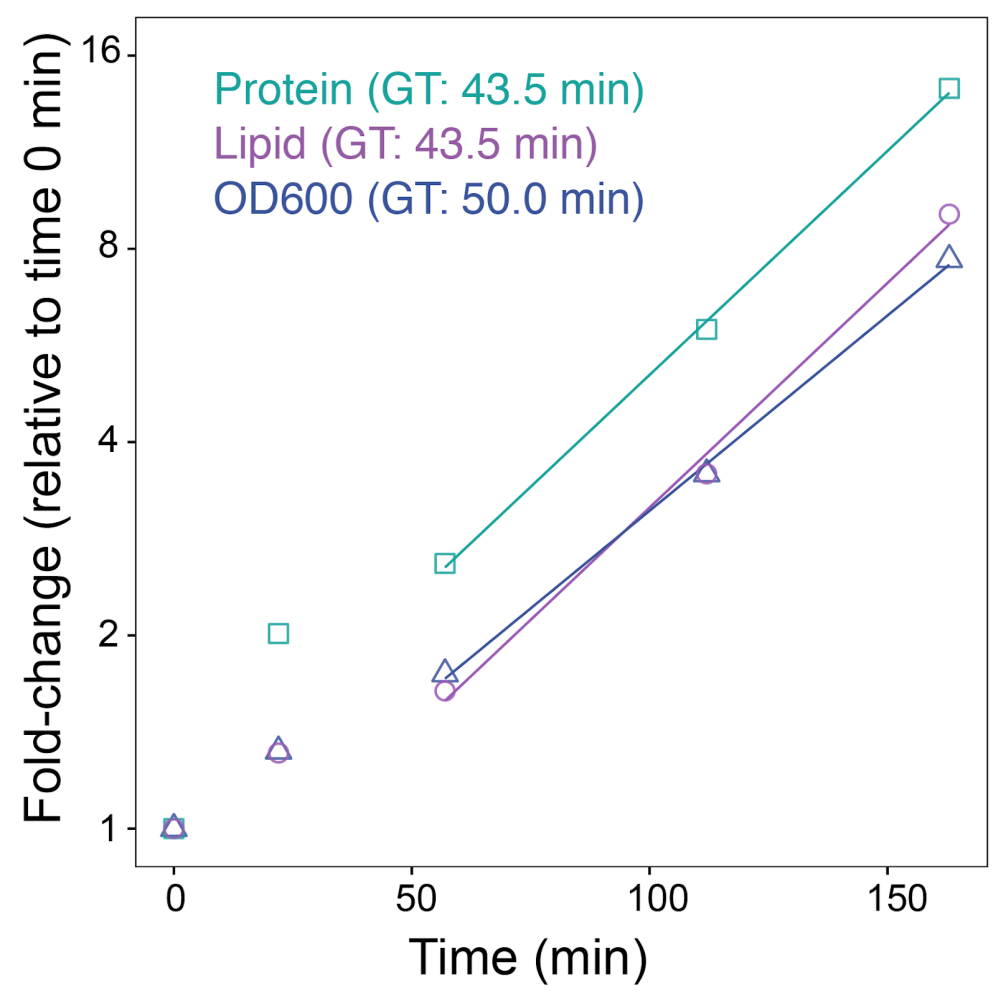

Figure S2: Estimates of generation time (GT) from lipid, protein and $\mathrm{OD}_{600}$ measurements are similar. Protein abundance grew disproportionately during initial cell expansion, likely owing to the fact that stationary phase cells were smaller than those in exponential phase. In exponential phase, the three methods yield generation times of 43.5 minutes for protein (green squares) as well as lipids (magenta circles) and 50 minutes for $\mathrm{OD}_{600}$ (blue triangles). Data from a single culture ( $u U$ culture) are shown. 


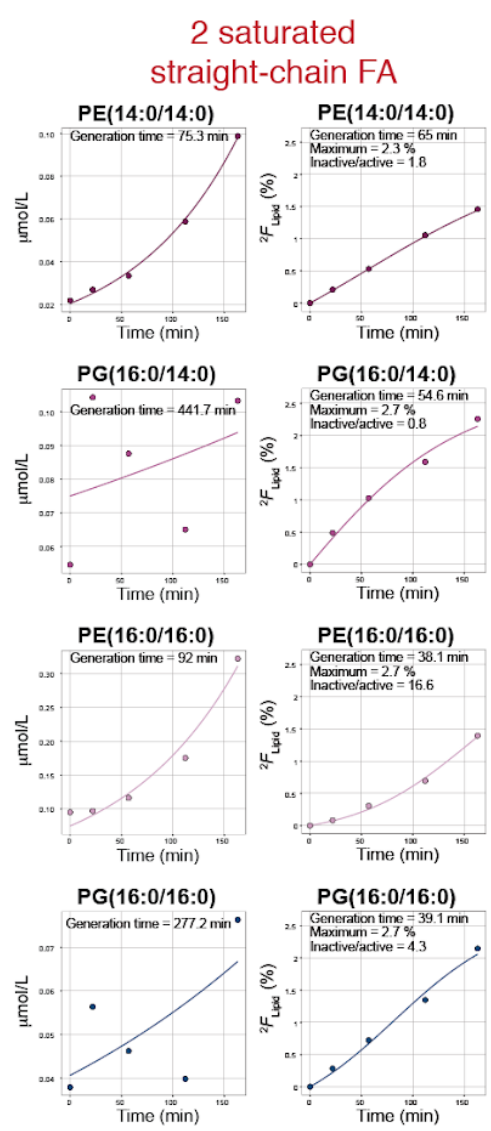

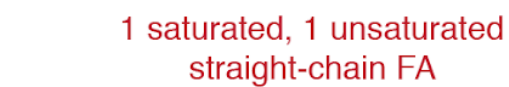
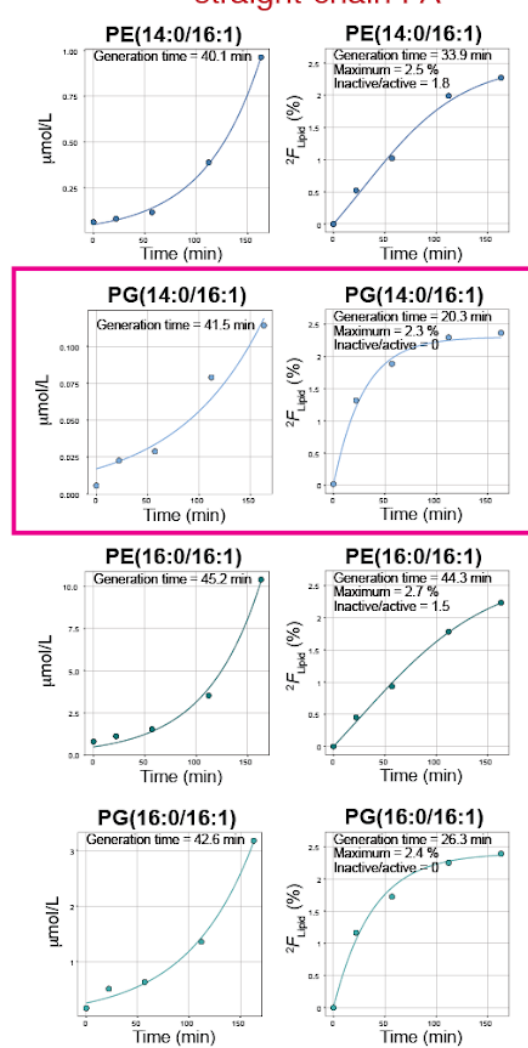

\section{(1)}
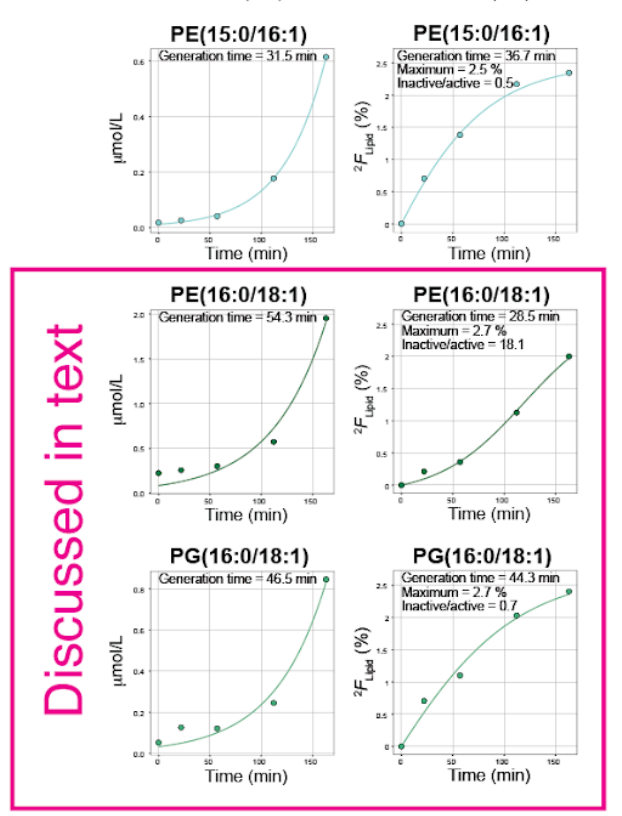

1 saturated straight-chain FA, 1 cyclopropane FA
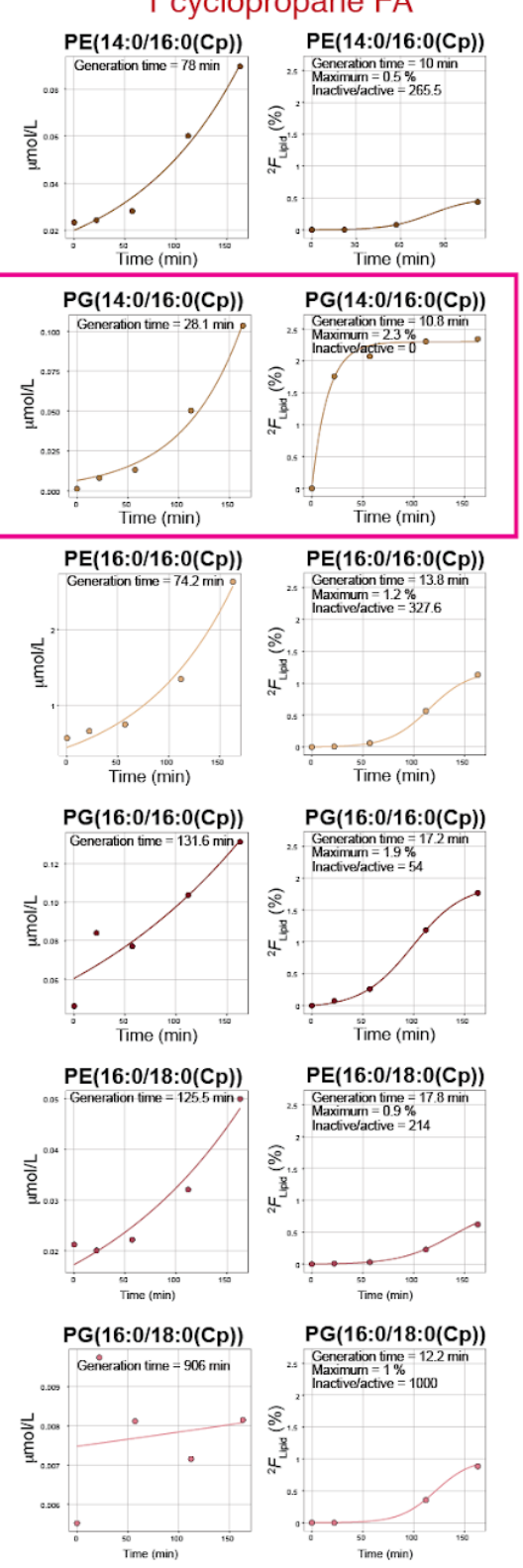

Figure S3: Overview of $E$. coli $P E$ and PG lipid production during the transition from stationary phase

into exponential phase. Phospholipids from culture $u L$ were quantified by the summed signals of their isotopologues and compared to their deuterium abundance $\left({ }^{2} F_{\text {LIPID }}\right)$. Lipids were grouped based on their fatty acyls, because this correlated with labeling dynamics. Notable exceptions of common trends were observed for example with $\mathrm{PE}(16: 0 / 18: 1)$ and $\mathrm{PG}(14: 0 / 16: 0(\mathrm{Cp}))$. 
bioRxiv preprint first posted online May. 25, 2018; doi: http://dx.doi.org/10.1101/330464. The copyright holder for this preprint (which was not peer-reviewed) is the author/funder, who has granted bioRxiv a license to display the preprint in perpetuity. It is made available under a CC-BY 4.0 International license.

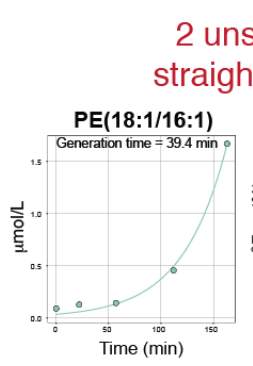

2 unsaturated straight-chain FA
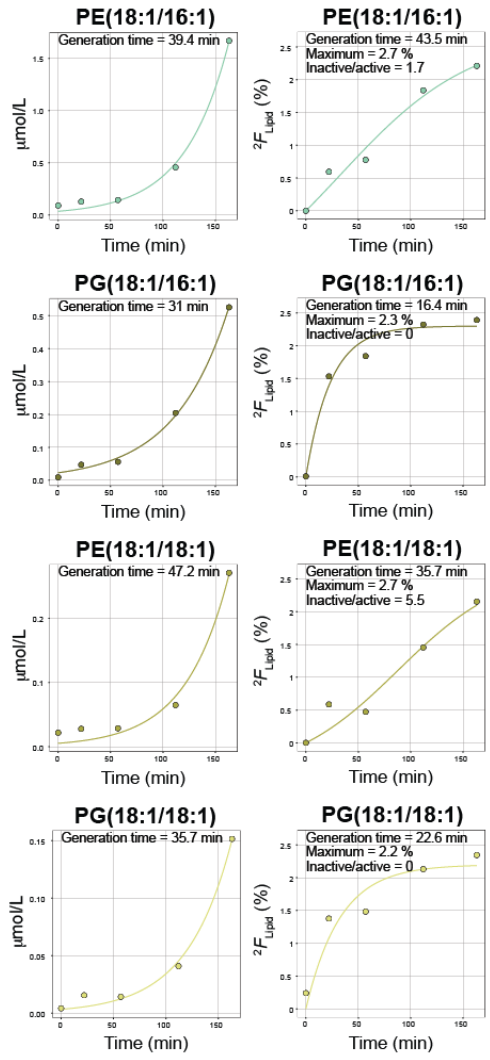

1 unsaturated straight-chain FA, 1 cyclopropane FA
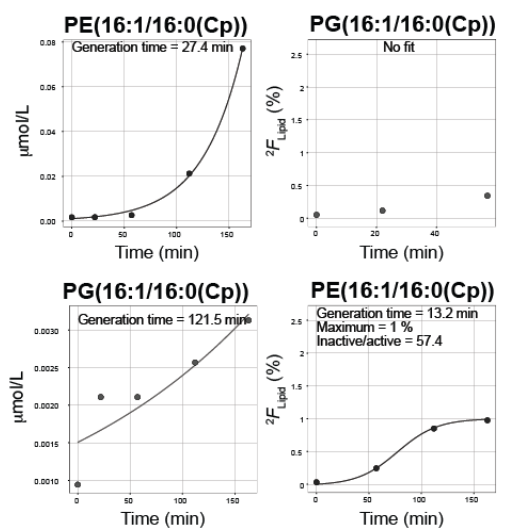

PE(16:1/16:0(Cp))
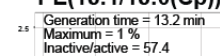

$\overbrace{}^{2}$

$4^{\frac{8}{3}}$
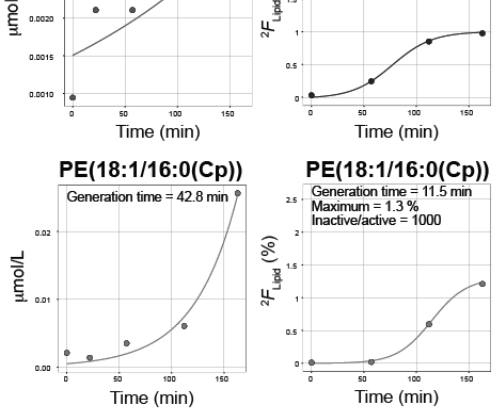

PE(18:1/16:0(Cp))

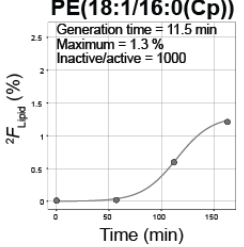

PG(18:1/16:0(Cp))

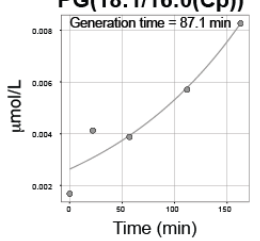

$P G(18: 1 / 16: 0(C p))$
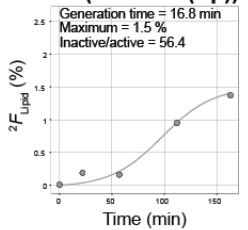

2 cyclopropane FA

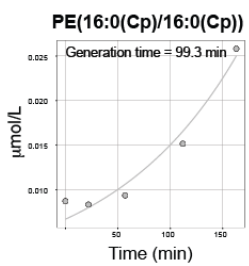

$\mathrm{PE}(16: 0(C p) / 16: 0(C p))$

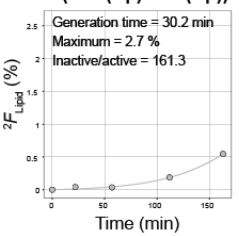

$\mathrm{PE}(18: 0(\mathrm{Cp}) / 16: 0(\mathrm{Cp}))$

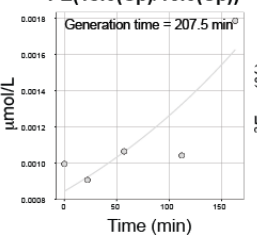

PE(18:0(Cp)/16:0(Cp))

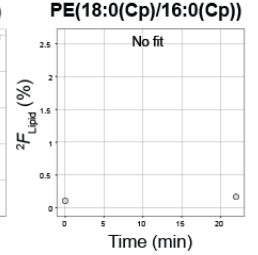

Figure S4: Overview of $E$. coli PE and PG lipid production during the transition from stationary phase

\section{into exponential phase (continued).}



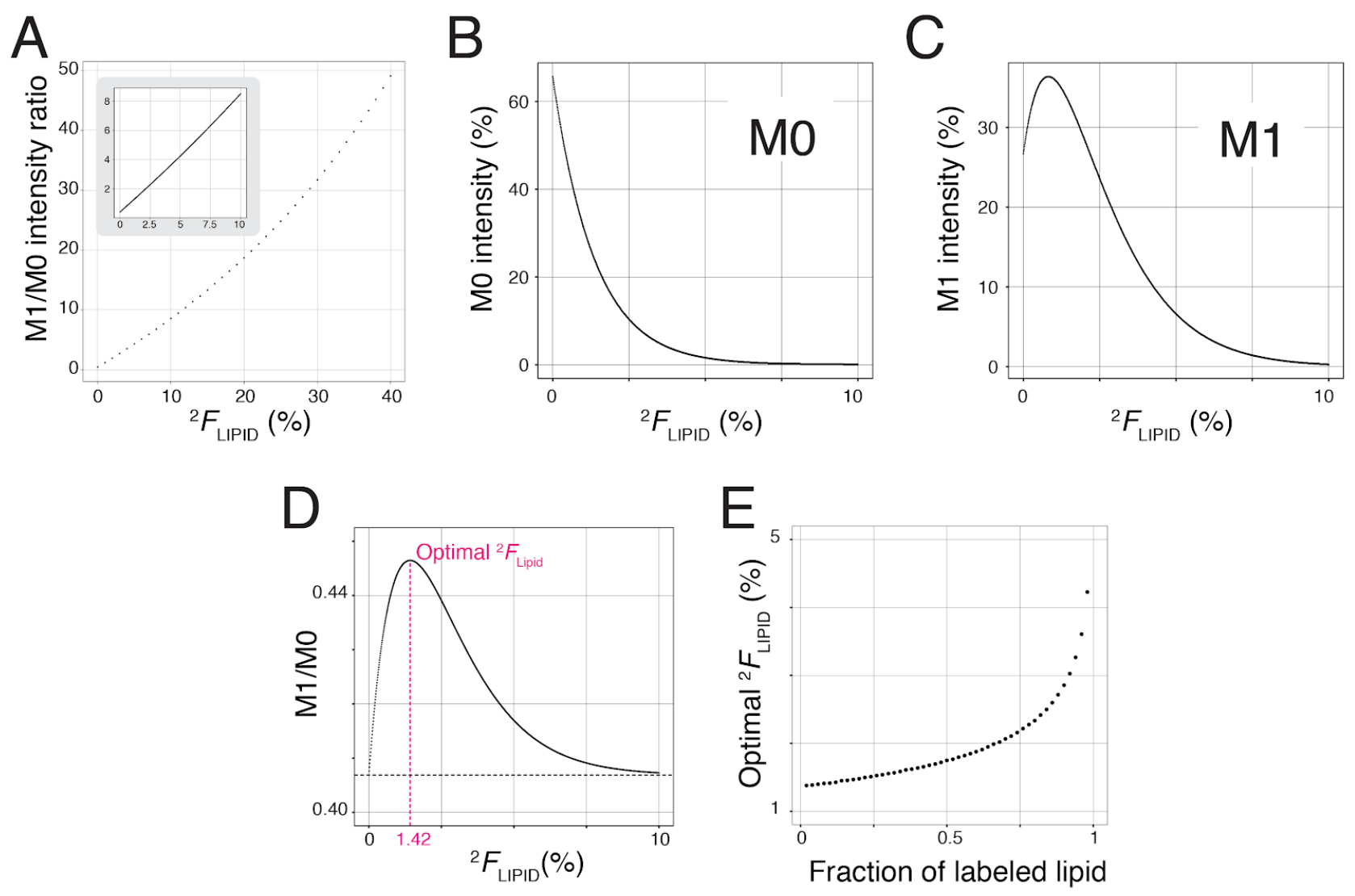

Figure S5: Deuterium content in lipids can be quantified using the ratio of M1 and M0 intensities. The sum formula of the phospholipid $\mathrm{PE}(16: 0 / 16: 1), \mathrm{C}_{37} \mathrm{H}_{73} \mathrm{NO}_{8} \mathrm{P}$, was used to model the behaviour of M1 and M0 isotopologues. (A) Deuterium abundance in lipid $\left({ }^{2} F_{\text {LIPID }}\right)$ was altered from 0 to 40 at\%. M1/M0 rises approximately linearly at ${ }^{2} F_{\text {LIPID }}<10$ at\% (inset). (B and C) Theoretical modeling of the proportion of M0 and M1 intensity compared to all isotopologues with varying ${ }^{2} F_{\text {LIPID }}$ (D) M1/M0 does not change linearly with increasing ${ }^{2} F_{\text {LIPID }}$ of labeled lipid when unlabeled material is also present. The calculation assumed a mixing of $90 \mathrm{~mol} \%$ unlabeled lipid and $10 \mathrm{~mol} \%$ deuterium labeled lipid of varying deuterium abundance ${ }^{2} F_{\text {LIPID }}$ (plotted on x-axis). In this scenario an optimum is observed when the labeled lipid portion has a ${ }^{2} F_{\text {LIPID }}$ value of $1.42 \%$. (E) This optimal ${ }^{2} F_{\text {LIPID }}$ increases the more labeled lipid is produced. When $2 / 3$ labeled lipid and $1 / 3$ unlabeled lipid is assumed, the optimal ${ }^{2} F_{\text {LIPID }}$ is about $2 \%$. The optimum also depends on the number of hydrogens in a lipid. 


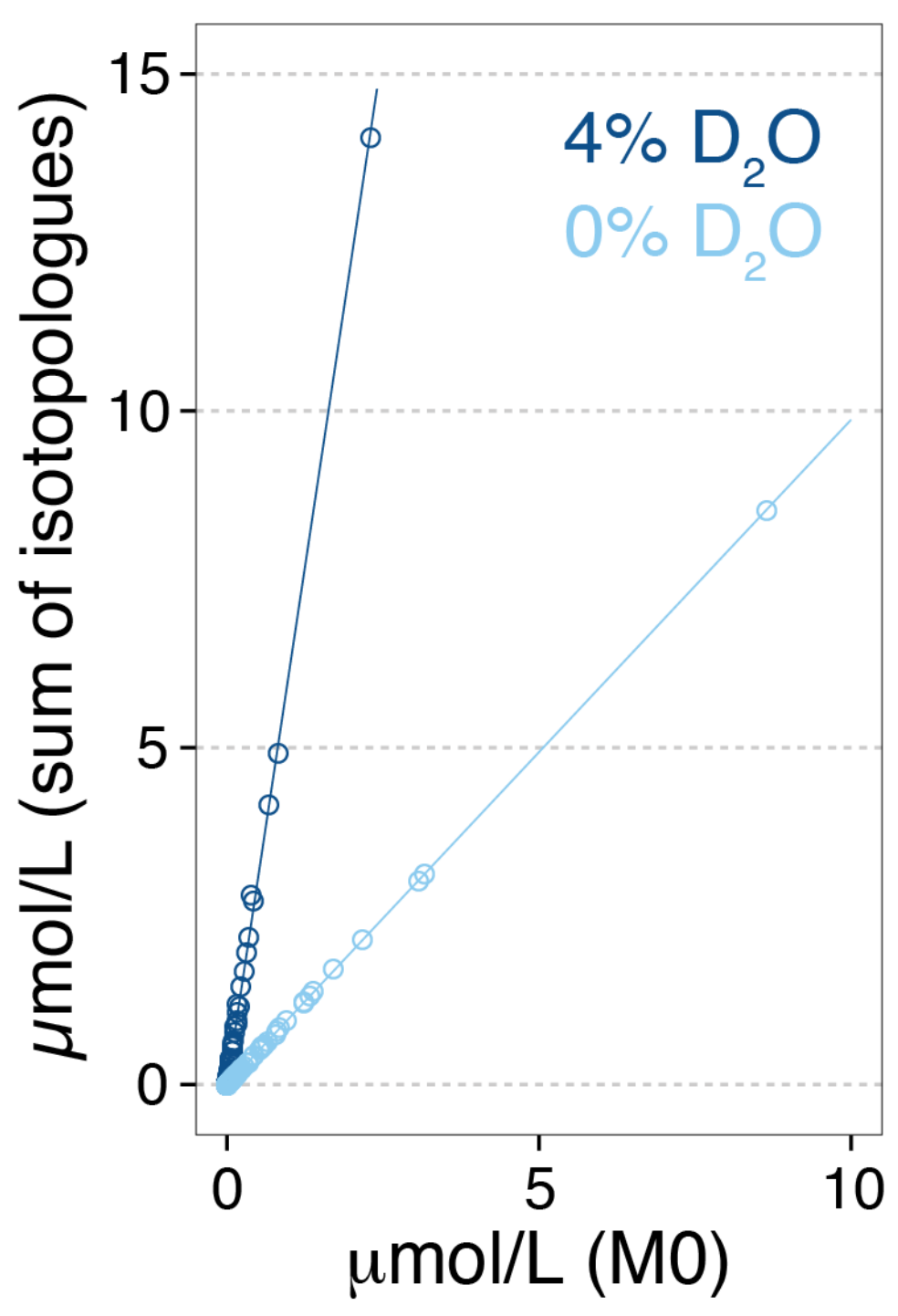

Figure S6: Absolute abundance of labeled lipids can quantified with the summed intensities of isotopologues. PE and PG lipids of two E. coli cultures unlabeled or full labeled in $4 \% \mathrm{D}_{2} \mathrm{O}$ medium, were quantified based on their monoisotopic signal or the sum of all isotopologues. A linear relationship between these two quantification methods were observed for lipids from both cultures. This suggests that, in experiments with little ion suppression, D-labeled lipids can be accurately quantified by comparing the sum 715 of isotopologue intensities of lipids and internal standards. In the unlabeled culture the slope was $0.977 \pm 0.034(1 \sigma)$ for PE, $0.980 \pm 0.037$ for $\mathrm{PG}$ and $1.05 \pm 0.118$ for $\mathrm{CL}$. 